\title{
For Want of a Nail: How Absences Cause Events
}

\author{
Phillip Wolff \\ Emory University
}

\author{
Aron K. Barbey \\ National Institutes of Health and Georgetown University
}

\author{
Matthew Hausknecht \\ Emory University
}

\begin{abstract}
Causation by omission is instantiated when an effect occurs from an absence, as in The absence of nicotine causes withdrawal or Not watering the plant caused it to wilt. The phenomenon has been viewed as an insurmountable problem for process theories of causation, which specify causation in terms of conserved quantities, like force, but not for theories that specify causation in terms of statistical or counterfactual dependencies. A new account of causation challenges these assumptions. According to the force theory, absences are causal when the removal of a force leads to an effect. Evidence in support of this account was found in 3 experiments in which people classified animations of complex causal chains involving force removal, as well as chains involving virtual forces, that is, forces that were anticipated but never realized. In a 4th experiment, the force theory's ability to predict synonymy relationships between different types of causal expressions provided further evidence for this theory over dependency theories. The findings show not only how causation by omission can be grounded in the physical world but also why only certain absences, among the potentially infinite number of absences, are causal.
\end{abstract}

Keywords: causation, causation by omission, causal models, knowledge structures, lexical semantics

Knowledge becomes more useful as it becomes more causal. Causal relations allow us to identify the factors that bring about events, diagnose problems, and predict the future. We know causal relations are vital, but in an important sense, we still do not know exactly what they are or exactly what it is people are pursuing when they seek a deeper causal understanding of a domain. One thing that is clear is that events sometimes occur due to the occurrence or appearance of another event or entity, a type of causation we refer to as positive causation (e.g., A causes B). Causal relations also hold between presences and absences (e.g., $\neg$ A causes B, A causes $\neg$ B), a type of causation we refer to as negative causation (Menzies, 2006). Although a unified account of causation should account for both positive and negative causation, present theories find it difficult to do so.

The difficulty appears most sharply with respect to a particular type of negative causation, causation by omission. Causation by omission is causation in which the absence of an influence brings about an effect, as in Not watering the plant caused it to wilt or Lack of clean air causes dizziness. This type of causation raises two general problems for theories of causation. First, there is the

Phillip Wolff and Matthew Hausknecht, Department of Psychology, Emory University; Aron K. Barbey, Cognitive Neuroscience Section, National Institute of Neurological Disorders and Stroke, National Institutes of Health and Department of Psychology, Georgetown University.

Matthew Hausknecht is now at the Department of Computer Science, University of Texas at Austin.

This research was supported in part by an award from the University Research Committee of Emory University.

Correspondence concerning this article should be addressed to Phillip Wolff, Emory University, Department of Psychology, 532 N. Kilgo Circle, Atlanta, GA 30322. E-mail: pwolff@emory.edu issue of how an absence can bring about an event in the first place. Theories that define causation in terms of the transmission or exchange of a conserved quantity-namely, process theories (Dowe, 2007) - are particularly vulnerable to this problem, because plainly, nothing can be transmitted from or exchanged with an absence (Craver, 2007; Menzies, 2006; Schaffer, 2000; Schulz, Kushnir, \& Gopnik, 2007; Woodward, 2006). The other salient issue with respect to causation by omission concerns what might be called the selection problem. Many factors in a situation may be necessary, and perhaps even sufficient, for bringing about an effect, but only one or a few of these factors will be construed as "the cause" of an event (Hesslow, 1988; Wolff \& Song, 2003). The selection problem poses a major challenge for dependency theories of causation, that is, theories that define causal relationships in terms of statistical, counterfactual, or logical diagnostics. As noted by philosophers, if causation by omission is actual causation, then there are many more candidate causes than common sense would allow (Beebee, 2004; Craver, 2007; McGrath, 2005). For example, consider a situation in which Peter did not put gasoline in his car, and ultimately his car stops. Most people would probably be willing to say that Peter's not putting gasoline in his car caused his car to stop; after all, there is a clear statistical/counterfactual dependency between Peter's nonaction and the engine's stopping: Had Peter put gas in the car, the car would not have stopped. However, if causation is defined in terms of dependencies, and dependencies can be established in terms of hypothetical events about what would have happened had somebody acted, then the number of possible causes becomes limitless. Peter is not the only person in the world who did not put gas in his car. There is also Susan, his cousin in Pennsylvania, Erik, Peter's best friend, the Pope, and the Queen of England, and so on. In the case of the Pope, it might be objected that he could not have been responsible 
because he was in Rome, but such an objection would, in fact, further highlight the depth of the problem. In dependency theories, a physical connection is not necessary for causation; all that is needed is a dependency.

Researchers have suggested that dependency theories might be able to handle the selection problem if they were augmented with certain restrictions, including constraints specified in terms of normality (Hart \& Honoré, 1985; McGrath, 2005) or various other legal, moral, or epistemic considerations (Beebee, 2005; Craver, 2007). Importantly, however, such criteria do not fall out of the theories, and their adoption would require new theories in their own right. An alternative strategy for trying to explain the phenomenon of causation by omission would be to adjust process theories. Such an approach is pursued in the present article. Specifically, we present a new process theory of causation, the force theory, which explains how causation by omission can be specified in terms of generative processes, but in a way that sharply restricts the range of causers. In three experiments, we used people's classifications of complex animations to test the force theory's prediction that causation by omission is instantiated by the removal or nonoccurrence of a suggested force. In a fourth experiment, we showed that the various correspondences between negative and positive causation that are entailed by all theories are better accounted for by the force theory than by dependency theories, indicating that a process approach to causation provides a more unified account of causation than do dependency theories.

\section{Dependency Theories}

In general, dependency theories hold that causal relations are specified in terms of the occurrence or nonoccurrence of events or states, without regard to the nature of the processes that produced those events or states (Ahn \& Kalish, 2000). For example, according to the probabilistic contrast model (Cheng \& Novick, 1992), causation is defined in terms of statistical dependencies. CAUSE relationships are implied when the probability of the effect in the presence of the cause, $\mathrm{P}(\mathrm{E} \mid \mathrm{C})$, is greater than the probability of the effect in its absence $\mathrm{P}(\mathrm{E} \mid \neg \mathrm{C})$, that is, $\mathrm{P}(\mathrm{E} \mid \mathrm{C})>\mathrm{P}(\mathrm{E} \mid \neg \mathrm{C})$. PREVENT relationships are implied when the inequality is in the opposite direction, $\mathrm{P}(\mathrm{E} \mid \mathrm{C})<\mathrm{P}(\mathrm{E} \mid \neg \mathrm{C})$.

Counterfactual theories of causation (Lewis, 1973) propose that $A$ causes $B$ holds if it is the case that if A had not occurred, then B would not have occurred (see also Lewis, 2000; Mackie, 1974; Mandel \& Lehman, 1996; Spellman, Kincannon, \& Stose, 2005; Spellman \& Mandel, 1999). A counterfactual criterion can presumably be extended to PREVENT relations (see Dowe, 2001) by negating the valence of the outcome. Specifically, as suggested by Walsh and Sloman (2009), A prevents $B$ holds if it is the case that if A had not occurred, then B would have occurred.

A third type of dependency theory is Goldvarg and JohnsonLaird's (2001) mental model theory, which characterizes not only CAUSE and PREVENT but also ALLOW. According to this theory, the notions of CAUSE, ALLOW, and PREVENT are associated with different combinations of possible co-occurrences, which are shown in Table 1. For example, a CAUSE relation is associated with a set of co-occurrences in which $\mathrm{A}$ is present and $\mathrm{B}$ is present; $\mathrm{A}$ is absent and $\mathrm{B}$ is present; and A is absent and $\mathrm{B}$ is absent.
Table 1

Possible Co-Occurrences Associated With the Concepts CAUSE, ALLOW, PREVENT

\begin{tabular}{llc}
\hline CAUSE & ALLOW & PREVENT \\
\hline $\mathrm{a} \mathrm{b}$ & $\mathrm{a} \mathrm{b}$ & $\mathrm{a} \neg \mathrm{b}$ \\
$\neg \mathrm{a} \mathrm{b}$ & $\mathrm{a} \neg \mathrm{b}$ & $\neg \mathrm{a}$ \\
$\neg \mathrm{a} \neg \mathrm{b}$ & $\neg \mathrm{a} \neg \mathrm{b}$ & $\neg \mathrm{a} \neg \mathrm{b}$ \\
\hline
\end{tabular}

Note. $\mathrm{a}=$ antecedent $\mathrm{b}=$ consequent $\neg=$ negation.

A fourth type of dependency theory is represented by Bayesian network theories of causation. In causal Bayesian networks, variables are connected to one another with "arcs," as in A $\rightarrow$ B. Each arc is associated with a set of conditional probabilities in conjunction with assumptions about the effects of actual or hypothetical interventions (Schulz et al., 2007; Sloman, 2005; Woodward, 2003, 2007). A recent account called the causal model theory shows how a Bayesian network approach to causation can be applied to the representation of CAUSE, ALLOW, and PREVENT, as well as causation by omission (Sloman, Barbey, \& Hotaling, 2009). A more detailed description of the causal model theory, as well as the other dependency theories, is provided in Appendix B.

Because mechanism does not play a role in dependency theories, they need not explain how an effect might be caused by an absence, thereby avoiding one of the problems raised by causation by omission. For example, in the probabilistic contrast model, causation by omission can be defined as present when the probability of the effect in the absence of the cause is greater than the probability of the effect in the presence of the cause, $\mathrm{P}(\mathrm{E} \mid \neg \mathrm{C})>$ $\mathrm{P}(\mathrm{E} \mid \mathrm{C})$. Although dependency theories offer accounts of causation by omission, as discussed earlier, they must all contend with the problem of how to curtail the essentially limitless number of possible absent causers that they allow for (Beebee, 2004; Craver, 2007; McGrath, 2005)

\section{Process Theories}

Process theories begin with the assumption that causation in the mind reflects the way events unfold in the physical world (Craver, 2007; Dowe, 2007; Machamer, Darden, \& Craver, 2000; Wolff, 2007). They hold that causation involves a transmission or exchange of physical quantities between the cause and the effect. For example, according to Aronson's (1971) transference theory, causation implies contact between two objects in which a quantity possessed by the cause (e.g., velocity, momentum, kinetic energy, heat, etc.) is transferred to the effect. Another transference theory is proposed by Fair (1979), who holds that causes are the source of physical quantities, energy, and momentum that flow from the cause to the effect. According to Salmon's $(1994,1998)$ invariant quantity theory, causation involves an intersection of world lines that results in the transmission of an invariant quantity. A recent proposal by Dowe (2000), the conserved quantity theory, breaks significantly from prior process theories by characterizing causal interactions as involving not a one-way transmission, but rather a bidirectional exchange of energy: Causal interactions occur when the trajectories of two objects (essentially, Salmon's "world lines") 
intersect, resulting in the exchange of conserved quantities (e.g., an exchange of momentum when two billiard balls collide).

The proposals just described come from philosophy. Similar proposals from psychology have been termed "generative theories" of causation. According to Bullock, Gelman, and Baillargeon (1982), adults believe that causes bring about their effects by a transfer of causal impetus. Shultz (1982) suggests that causation is understood as a transmission between materials or events that results in an effect. According to Leslie (1984), physical causation is processed by a "theory of bodies" that schematizes objects as bearers, transmitters, and recipients of a primitive notion of force.

As noted earlier, the main criterion for causation in process theories, the transfer or exchange of energy or force, is clearly at odds with causation by omission (Craver, 2007). For example, when we say Lack of rainfall causes crops to fail, the cause in this claim, Lack of rainfall, is an absence, utterly lacking in force. The problem posed by causation by omission has led some philosophers and psychologists to propose that there must be two kinds of causation, one based on the transmission of energy or force and another based on dependencies that can be used for the representation of negative causation (Godfrey-Smith, in press; Hall, 2004; Lombrozo, 2009; Menzies, 2006). Other philosophers have argued that causation by omission is not "really" causation (Beebee, 2004; Dowe, 2001). For example, Dowe (2001) views causation by omission as "quasi" causation because it does not involve an exchange of conserved quantities and accounts for statements of causation by omission by adopting theoretical machinery from counterfactual theories. For other theorists, the inability of process theories to account for causation by omission indicates that such theories are fundamentally flawed (Craver, 2007; Menzies, 2006; Schaffer, 2000; Schulz et al., 2007; Woodward, 2006).

Although the phenomenon of causation by omission clearly conflicts with the assumptions of past process theories, we submit that the problem has more to do with the way such theories have been implemented than with the assumption that people specify causation in terms of processes. Below we sketch out in general terms how causation by omission might be handled by a process approach to causation by loosening the restriction that causation must involve a transmission or exchange of energy between the initial cause and final effect. We then show how this solution can be formalized in a new theory of causation, the force theory.

\section{Causation by Omission as Based on Force Removal}

We propose that absences are causal when the removal or nonrealization of an anticipated force leads to an effect. To illustrate this idea, consider a situation in which a car is held off the ground by a jack. A man pushes the jack aside-removing the force holding up the car-and the car falls to the ground. This situation instantiates a type of causation by omission, as indicated by the acceptability of the description The lack of a jack caused the car to fall to the ground. We further note that the removal of a force occurs within the context of a series of PREVENT relations involving three entities/forces (e.g., those of the man, the jack, the car); such a sequence of events is often referred to as double prevention (Collins, 2000; Dowe, 2001; Hall, 2000, 2004). Initially, the force of the jack is preventing the car from falling to the ground; the man then prevents the jack from preventing the car from falling. Combining these two PREVENT relations, we can establish a relationship between the man and the car, specifically, The man caused the car to fall to the ground. Thus, our proposal is that causation by omission is always embedded within a double prevention and that it names the relationship between the second and third entities involved. In double preventions, the second entity is removed, and so the relationship between the second and third entities concerns what happens to the third entity in the absence of the second entity.

Our proposal handles the problem of causation by omission by relaxing the constraint that causation entails the transmission of force from the first to the final entity in the causal chain. In the above example, the force generated by the man is not transmitted to the car but rather serves to remove the force that is holding up the car. Thus, although there is a local transfer of force between the first pair and between the last pair of entities, force is not transferred from the first to the last entity.

In accounting for causation by omission, our proposal places limits on the range of absences that can be causal. In our approach, absences are not total absences; they are forces that at one point had an effect on one of the entities but were removed from the interaction due to another force. In this way, the force theory sharply constrains the range of possible absences that might give rise to an effect, thereby addressing the selection problem.

The link between the interaction of forces in the world and categories of causal relations is formalized in the force theory described below. We offer this formalization in order to show that there are no mysterious quantities in our account, that the notion of causation by omission can be fully grounded in objective, measurable properties in the world. First, we explain how the theory represents individual causal relationships. Then, we show how the theory, via relation composition, accounts for the joining of causal relations that allow for the representation of double preventions. Finally, we describe how the theory accounts for the representation of causation by omission and causation of an absence.

\section{The Force Theory}

The force theory generalizes Wolff's (2007) dynamics model of causation, which is based on Talmy's (1988) theory of force dynamics. Individual interactions involve two main entities: an affector and a patient (the entity acted on by the affector). The force theory holds that people specify causal relations in terms of configurations of forces that are evaluated with respect to an endstate, that is, a possible result state. The forces may be physical, psychological (e.g., intentions), or social (e.g., peer pressure) (Wolff, 2007). We assume that people's representations of the forces in physical events are relatively accurate with respect to their direction, but not magnitude, because most physical situations are compatible with a wide range of force magnitudes, and determining exact magnitudes is often impossible without explicit measurements. Uncertainty about the magnitudes of forces implies that a certain level of indeterminacy is built into people's representations of causation. Nevertheless, because their representations of the forces are relatively accurate with respect to direction, it is assumed that people are able to conduct partial "reenactments" of the processes that join forces in the world. A reenactment involves specifying the objects and the forces acting on those objects in a situation. It also involves carrying out a simulation showing what 
happens as a consequence of the forces acting on the objects. Causal reasoning is assumed to consist of such reenactments.

\section{Representing Individual Causal Relations}

A theory of how people represent individual causal relations is specified in the dynamics model (Wolff, 2007). According to the dynamics model, causal concepts are specified in terms of three dimensions: (a) the tendency of the patient for an endstate, (b) the presence or absence of concordance between the affector and the patient, and (c) progress toward the endstate (essentially, whether or not the result occurs). Table 2 summarizes how these dimensions differentiate the concepts of CAUSE, ALLOW, and PREVENT. For example, when we say "High winds caused the tree to fall," we mean that the patient (the tree) had no tendency to fall (tendency $=$ No), the affector (the wind) acted against the patient (concordance $=\mathrm{No}$ ), and the result (falling) occurred (endstate approached $=$ Yes).

The dynamics model specifies how these three dimensions are captured in terms of configurations of force vectors. Sample configurations for CAUSE, ALLOW, and PREVENT are depicted in Figure 1. As is customary, the free-body diagrams in Figure 1 show forces acting on only one object, the patient entity. They do not show the location of the affector entity, only the direction and magnitude of the affector's force on the patient (i.e., A). Similarly, they do not show the location of the endstate, just the direction the patient must move in order to reach the endstate (i.e., E). In each of the configurations shown in Figure 1, the patient entity is also associated with a force (i.e., P). If the patient were a boat, for instance, the patient force would correspond to the force generated by the boat's motor. When the patient has a tendency for the endstate, the patient vector, $\mathbf{P}$, will point in the same direction as the endstate vector, $\mathbf{E}$; otherwise, $\mathbf{P}$ will point in a different direction. When the patient and the affector are in concordance, their respective vectors will point in the same direction. Finally, the patient entity will approach the endstate when the resultant (sum) of the $\mathbf{A}$ and $\mathbf{P}$ vectors, $\mathbf{R}$, is in the same direction as the endstate vector, $\mathbf{E}$.

Support for the dynamics model's account of CAUSE, ALLOW, and PREVENT was provided in a series of experiments in which participants categorized 3-D animations of realistically rendered objects with trajectories that were wholly determined by the force vectors entered into a physics simulator. As reported in Wolff (2007; Wolff \& Zettergren, 2002), people's descriptions of the events closely matched the predictions of the model.

\section{Combining Relations in the Force Theory}

Whereas the dynamics model accounts for how people represent individual relations, the force theory specifies how people combine

Table 2

Representations of Several Causal Concepts

\begin{tabular}{lccc}
\hline \multicolumn{1}{c}{ Concept } & $\begin{array}{c}\text { Patient tendency } \\
\text { for endstate }\end{array}$ & $\begin{array}{c}\text { Affector-patient } \\
\text { concordance }\end{array}$ & $\begin{array}{c}\text { Endstate } \\
\text { approached }\end{array}$ \\
\hline CAUSE & No & No & Yes \\
ALLOW (also HELP, & Yes & Yes & Yes \\
ENABLE) & Yes & No & No \\
PREVENT & & & \\
\hline
\end{tabular}

them to generate new relations through the process of relation composition. For example, given the relations nerve damage causes pain and pain causes lost workdays, people may derive the overarching relation nerve damage causes lost workdays. Relation composition is essential to causation by omission because it is assumed to comprise a series of PREVENT relations. Below, we explain how relation composition is accomplished.

In the force theory, the mechanism for combining relations depends on whether the initial relation in a pair of relations is generative (i.e., CAUSE or ALLOW) or preventive. When the initial relation is generative, the resultant of the initial relation becomes the affector in the second relation. The idea can be illustrated by a multiple-collision event like the one shown in Figure 2. In this sequence of events, A begins moving first, it hits $\mathrm{B}$, and $\mathrm{B}$ then hits $\mathrm{C}$, sending $\mathrm{C}$ over the line. The arrows in Figure 2 indicate the directions of the cars' motion. Cars without arrows are not moving.

Figure 3 illustrates the forces involved in the animation depicted in Figure 2. On the left side of Figure 3 is a picture of the first frame of the animation. A's force on B is indicated by the longest vector pointing to the right immediately above $B$. This is the affector vector acting on $\mathrm{B}$. Notice that the direction of the affector vector is in the same direction as the front of $\mathrm{A}$. B resists moving in the direction of the force from A due to friction. This resistance is indicated by the vector pointing to the left immediately above B (the vector magnitudes are not drawn to scale). Notice that B is pointed in the same direction as the friction vector, which constitutes B's tendency. The resultant of the forces associated with these two vectors is the vector with the dotted line, which points to the right. This resultant force propels B into C. B's force on C is indicated by the longest vector pointing to the right immediately above $\mathrm{C}$. This force was originally the resultant of the forces acting on B. Like B, C resists moving in the direction of the affector vector due to friction. This resistance is indicated by the small vector pointing to the left. The resultant of these two vectors is the dotted vector immediately above $\mathrm{C}$, which propels $\mathrm{C}$ over the line.

On the right side of Figure 3 is a pair of free-body diagrams depicting the configurations of forces instantiated in the single frame of the animation arranged vertically. Given that any number of causal relations can be added together, the forces are labeled alphabetically down the causal chain as needed. Resultant vectors are labeled by combining the two letters and reversing their alphabetic order. The free-body diagram at the top depicts the configuration of forces acting on $\mathrm{B}$. The free-body diagram below depicts the configuration of forces acting on $\mathrm{C}$. The vertical arrow connecting the resultant vector in the first configuration with the affector vector in the second configuration is there to highlight that the resultant from the first configuration is transferred to the second configuration. In this particular animation, the two configurations of forces constitute CAUSE relations. As discussed earlier, when the initial relation is generative (e.g., a CAUSE relation), force is transmitted through the chain by using the resultant vector of the first configuration of forces as the affector force in the following configuration.

When the first relation in a pair of premises is preventive (e.g., A PREVENT B), relation composition proceeds differently. Note that if all of the forces are actual (not virtual), then if A first prevents B, B cannot act on C (because B has already been prevented). The way in which such chains are understood, then, is 

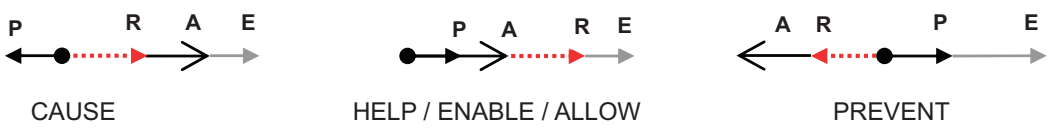

Figure 1. Configurations of forces associated with CAUSE, HELP/ENABLE/ALLOW, and PREVENT; $\mathbf{A}=$ the affector force; $\mathbf{P}=$ the patient force; $\mathbf{R}=$ the resultant force; $\mathbf{E}=$ endstate vector, which is a position vector, not a force.

that a PREVENT relation must first exist between $\mathrm{B}$ and $\mathrm{C}$, and then A can act on B in such a way that B's force is removed. The intuition behind this can be illustrated with a real-world example of double prevention, namely, pulling a plug to allow water to flow down the drain. This sequence of PREVENTS begins with the plug (B) preventing the water (C) from draining (that is, the second premise in a double prevention). Then, someone (A) prevents B by pulling the plug, that is, removing B's force on C. Note that when A pulls B, A opposes not just the force associated with $\mathrm{B}$, but also the force associated with $\mathrm{C}$, that is, the resultant of the $\mathbf{B}$ and $\mathbf{C}$ forces (the plug and the water). Thus, in the case of double prevention, the resultant of the second premise (CB), which is computed first, serves as the patient vector in the first premise $\left(\mathbf{B}_{\mathbf{C B}}\right)$.

The way forces are transmitted in a double prevention can be illustrated in a different way based on the chain depicted in Figure 4. In the beginning of the animation depicted in Figure 4, $\mathrm{C}$ approaches the line. $\mathrm{B}$ then approaches $\mathrm{C}$ and prevents it from crossing the line. The middle panel shows A pulling B away. In the panel on the far right, with the removal of $\mathrm{B}, \mathrm{C}$ crosses the line. The forces involved in the animation shown in Figure 4 are depicted in Figure 5.

On the left side of Figure 5 is a picture of the first frame of the animation. The long arrow above $\mathrm{C}$ (pointing to the left) represents the force imparted on $\mathrm{C}$ by $\mathrm{B}$, whereas the short arrow above $\mathrm{C}$ (pointing to the right) represents C's tendency to cross the line. The resultant force acting on $\mathrm{C}$ - the dotted arrow pointing to the left-prevents $\mathrm{C}$ from continuing to move toward the line. The long arrow above B (pointing to the right) represents the force imparted on it by A, whereas the short arrow above B represents the resultant of the forces acting on $\mathrm{C}$. Note that the force of $\mathrm{A}$ acting on B does not oppose the force associated with B alone. The force from $\mathrm{A}$, in a sense, gets some help in moving $\mathrm{B}$ from the force $\mathrm{C}$ imparts on B. Hence, the force from A opposes the resultant of the forces associated with $\mathrm{B}$ and $\mathrm{C}$.

Immediately to the right of the picture in Figure 5 is a pair of free-body diagrams depicting the same configurations of forces shown in the frame of the animation, this time arranged vertically.

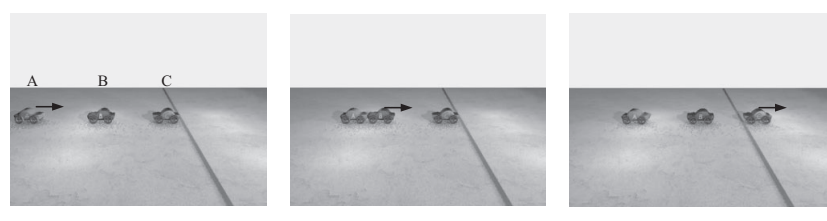

CAUSE/CAUSE (A/B caused $C$ to cross the line.)

Figure 2. The animation begins with all of the cars stationary. A begins moving first. It hits $\mathrm{B}$, sending $\mathrm{B}$ into $\mathrm{C}$, which then moves over the line. The animation can be summarized by the sentence $A$ caused $C$ to cross the line.
The free-body diagram at the top depicts the configuration of forces acting on $\mathrm{B}$. The free-body diagram below depicts the configuration of forces acting on $\mathrm{C}$. The vertical arrow connecting the resultant vector in the second configuration with the patient vector in the first configuration highlights the fact that the resultant is transferred from one configuration to the next. As discussed above, in chains of PREVENT relations, the resultant of the second PREVENT configuration serves as the patient vector in the first PREVENT configuration.

\section{Generating a Conclusion}

Regardless of how the force configurations are combined, the manner in which an overall conclusion is generated is the same. As depicted in Figure 6, the affector in the conclusion is the affector from the first premise (A); the endstate in the conclusion is the endstate from the previous premise $(\mathbf{E})$; and the patient in the conclusion is the resultant of the patient vectors in the premises $(\mathbf{B}+\mathbf{C})^{1}$

\section{ALLOW Relations}

In the preceding discussion, we reviewed how double preventions entail the removal of a force and, as a consequence, how they may underlie people's representations of causation by omission. Following the lead of McGrath (2003), we propose that double preventions can be expressed not only as causations by omission, in which the affector is absent, but also as ALLOW and CAUSE relations in which an affector is present. For example, consider again the double prevention involved in pulling a plug and letting water run down a drain. One way we could describe the event is in terms of causation by omission: lack of a plug allowed the water to drain. Alternatively, we could describe the event in terms of the entity that prevented the prevention: Jack allowed the water to drain or Jack caused the water to drain (by pulling the plug). In other words, double preventions can be described in terms of either

\footnotetext{
${ }^{1}$ As noted earlier, according to counterfactual theories of causation, the statement $A$ causes $C$ holds if it is the case that if A had not occurred, $\mathrm{C}$ would not have occurred. Such counterfactuals can be evaluated using vector representations. To simulate the event of what would have happened if A had not occurred, all that we need to do is sum all of the vectors in the causal chain, except for the A vector, and then compare this vector with the endstate vector to determine whether the result occurs. Such a resultant is the same as the patient vector in the conclusion as specified by the force theory; hence, the patient vector in the conclusion makes it possible to evaluate the counterfactual that, according to many theories, is essential to determining causation (see Lewis, 1973, 2000).
} 

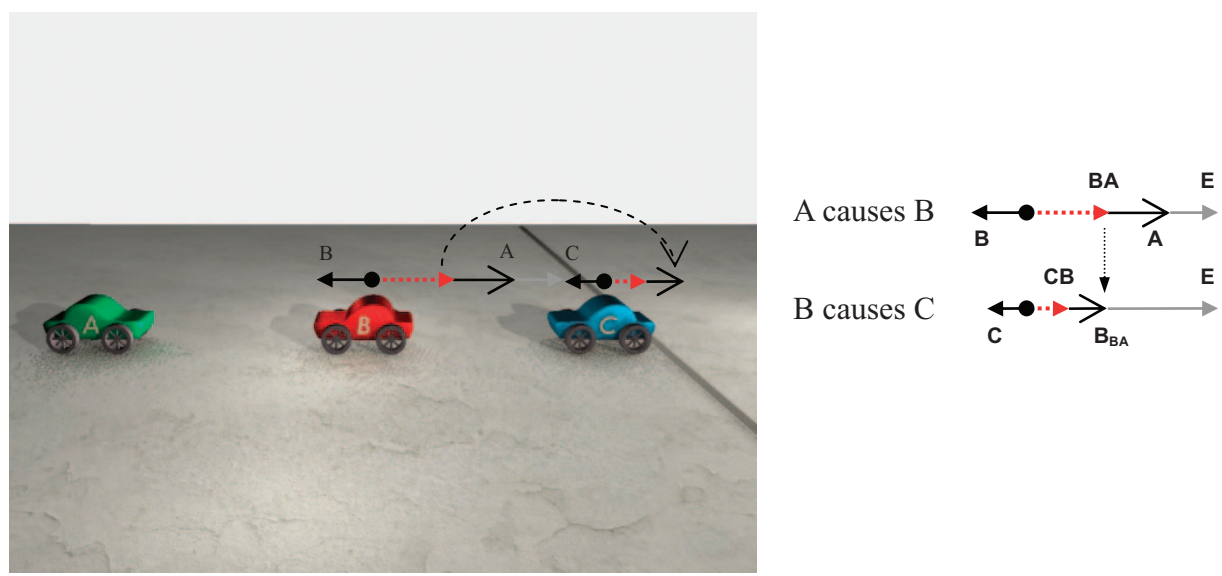

Figure 3. On the left side is a frame from an animation in which A bumps B, and then B bumps C. Above cars $\mathrm{B}$ and $\mathrm{C}$ are free-body diagrams. The smaller vectors pointing to the left are the patient vectors acting on $\mathrm{B}$ and $\mathrm{C}$ (i.e., friction). The longer vectors pointing to the right are affector vectors, and the dashed vectors are the resultant vectors. Note that in this sequence of collisions, the resultant vector associated with B becomes the affector vector acting on $\mathrm{C}$. On the right side are two free-body diagrams depicting the same forces shown on the left side, but this time, the configuration of forces are arranged vertically rather than horizontally. In the free-body diagrams, the vector $\mathbf{E}$ is the position vector pointing to the endstate, which, in the animation, is the area on the right side of the line.

the force that is removed (i.e., the lack of the plug) or the removing force (i.e., Jack). Similarly, in the animation depicting double prevention in Figures 4 and 5, we can say either the absence of car B allowed car C to cross the line or the presence of car A allowed car $\mathrm{C}$ to cross the line. Following prior work in philosophy (Foot, 1967; McGrath, 2003; McMahan, 1993), psychology (Barbey \& Wolff, 2006, 2007, 2009; Chaigneau \& Barbey, 2008; Sloman et al., 2009; Wolff, 2007), and linguistics (Talmy, 1988), we propose that the concept of ALLOW is necessarily based on double prevention.

\section{Accounting for Multiple Conclusions}

In the force theory, relation composition can sometimes give rise to more than one conclusion. The reason why is because the relations in a chain can be instantiated by many different sets of forces. When added together, the magnitudes of the forces in one instantiation might produce one conclusion, whereas the magnitudes of the forces in another instantiation might produce another conclusion. Double preventions are one type of chain in which different instantiations of forces can lead to different conclusions. As argued by several researchers, the composition of two
PREVENT relations sometimes leads to CAUSE relations and other times to ALLOW relations (Barbey \& Wolff, 2006, 2007, 2009; Chaigneau \& Barbey, 2008; McGrath, 2003; Sloman et al., 2009). The force theory allows for both of these possible interpretations, as illustrated by the pair of configurations shown in Figure 7.

As shown on the left side of Figure 7, in some double preventions, the two patient vectors in the premises combine to produce a patient vector in the conclusion $(\mathbf{C})$ that points toward the endstate, resulting in an ALLOW configuration. However, as shown on the right side of Figure 7, the patient vectors in a double prevention can also sometimes combine to produce a patient vector in the conclusion that points away from the endstate, resulting in a CAUSE configuration.

Whereas a chain of relations can give rise to more than one conclusion, the force theory constrains the percentage of times a particular conclusion will arise for a particular causal chain. One way these percentages can be determined is to systematically vary the magnitudes of the forces in a causal chain so that all possible combinations of force magnitudes are examined. A program has been written that conducts such a process and then counts the
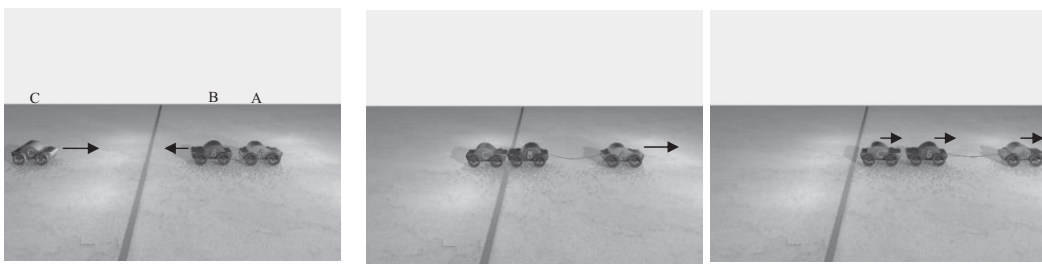

Figure 4. The still frames depict key stages in a PREVENT/PREVENT chain. First, $\mathrm{C}$ attempts to cross the line but is prevented by B. Then, A pulls B away from $\mathrm{C}$ with a rope, preventing B from preventing $\mathrm{C}$. With the removal of $\mathrm{B}, \mathrm{C}$ crosses the line. 

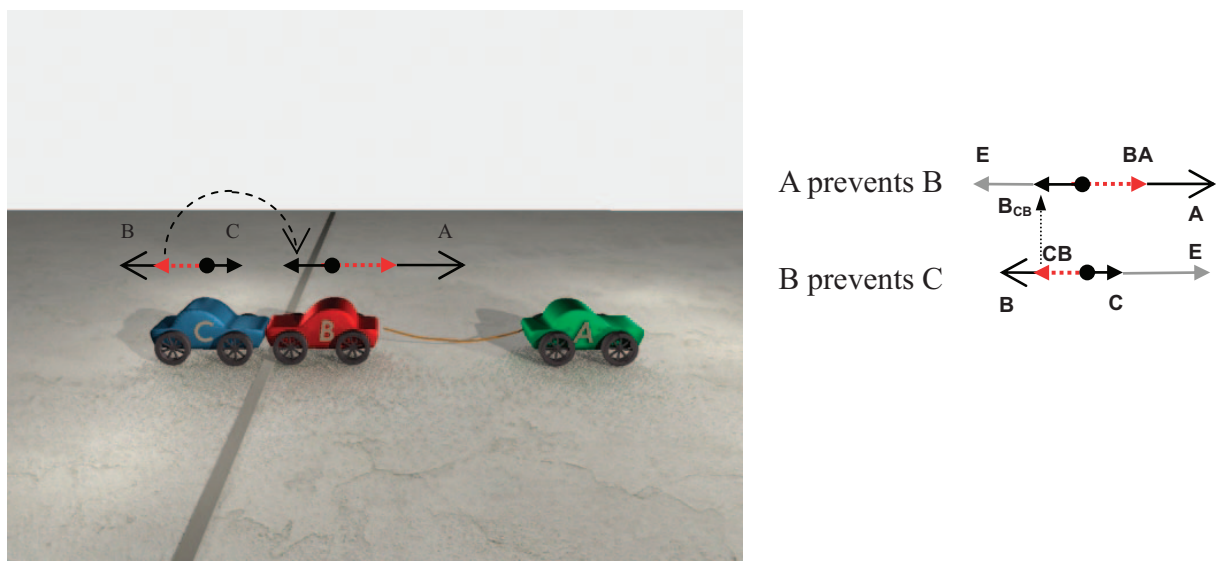

Figure 5. On the left side is a frame from an animation in which B prevents $\mathrm{C}$ from crossing a line, and then A pulls $\mathrm{B}$ away from $\mathrm{C}$, and $\mathrm{C}$ is able to cross the line. Above cars $\mathrm{B}$ and $\mathrm{C}$ are free-body diagrams showing the forces acting on these cars. The smaller vectors pointing left and right are patient vectors, whereas the longer vectors are affector vectors. The dashed vectors are resultant vectors. On the right side are two free-body diagrams depicting the same forces shown on the left, but this time, the configuration of forces are arranged vertically rather than horizontally. In the free-body diagrams, the vector $\mathbf{E}$ is the position vector pointing to the endstate, which, in the animation on the left, is the area on the right side of the line. Note that in a double prevention, the resultant vector of $\mathrm{B}$ and $\mathrm{C}$ becomes the patient vector in the interaction between $\mathrm{B}$ and $\mathrm{A}$.

number of times a particular conclusion is generated (http:// userwww.service.emory.edu/ pwolff/Transitivedynamics.htm). In effect, the program computes the area under the probability distribution implied by a particular causal chain. As explained in detail in Barbey and Wolff (2009), the results from this program can also be computed using integral calculus. With respect to double preventions, in particular, the program (as well as integral calculus) indicates that such chains will lead to ALLOW conclusions $62 \%$ of the time and to CAUSE conclusions $38 \%$ of the time.

As explained in Barbey and Wolff (2009), double preventions are just one among a number of causal chain types that are predicted to give rise to more than one conclusion. For example, the composition CAUSE $\circ A L L O W$ is predicted to lead to ALLOW conclusions $76 \%$ of the time and CAUSE conclusions $24 \%$ of the time, whereas the composition PREVENT $\triangle$ CAUSE is predicted

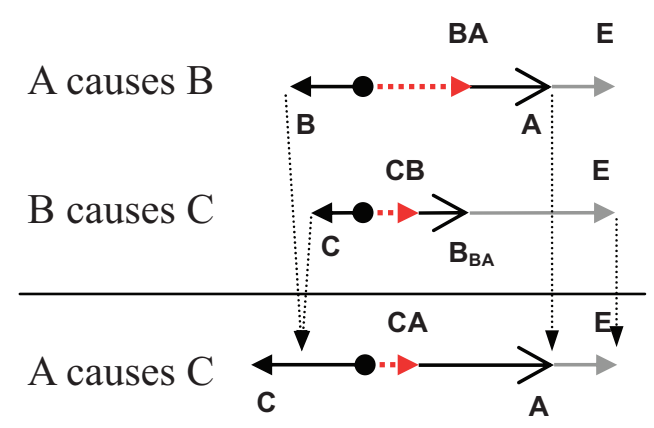

Figure 6. The affector force in the conclusion, $\mathbf{A}$, is the affector force in the first relation, $\mathbf{A}$. The endstate in the conclusion is the endstate vector from the previous premise. The patient force in the conclusion, $\mathbf{C}$, is based on the vector addition of the patient forces $\mathbf{B}$ and $\mathbf{C}$ in the premises. to lead to CAUSE conclusions $49 \%$ of the time and ALLOW conclusions $22 \%$ of the time. For a number of other compositions, only one conclusion is predicted. For example, the composition CAUSEOPREVENT is predicted to give rise to PREVENT conclusions $100 \%$ of the time, and the composition ALLOW०ALLOW is predicted to lead to ALLOW conclusions $100 \%$ of the time. In yet other relation compositions, the theory still predicts only one conclusion, but at a weaker level. For example, the composition PREVENT ${ }^{\circ} \mathrm{CAUSE}$ gives rise to PREVENT conclusions $37 \%$ of the time. The remaining $63 \%$ of the conclusions are associated with an undefined configuration of forces. Under these conditions, we predict that people would associate a PREVENT ${ }^{\circ}$ CAUSE composition with a PREVENT conclusion, but to a weaker degree than, for example, a CAUSE PREVENT composition, which gives rise to a PREVENT conclusion $100 \%$ of the time.

\section{Representing Causation of Absences}

The preceding discussion focused on causation by omission, that is, causation typically expressed in the form $\neg A$ causes $B$. Another type of negative causation is causation of an absence, which is typically expressed in the form $A$ causes $\neg B$, as exemplified by the sentences Pain causes lack of sleep and Black holes allow no escape. We propose that people represent causation of an absence by treating the negation of the consequent as a PREVENT relation in a causal chain. The PREVENT relation is added to the causal chain by assuming an unnamed entity to connect the relations that can be referred to by $x$. Expressions of the form $A$ causes $\neg B$ are thereby represented as A CAUSES $\mathrm{x}, \mathrm{x}$ PREVENTS $\mathrm{B}$. The overarching relation implied by this causal chain is based on the relation composition of CAUSE and PREVENT relations, which, according to the force theory, is a PREVENT relation virtually 

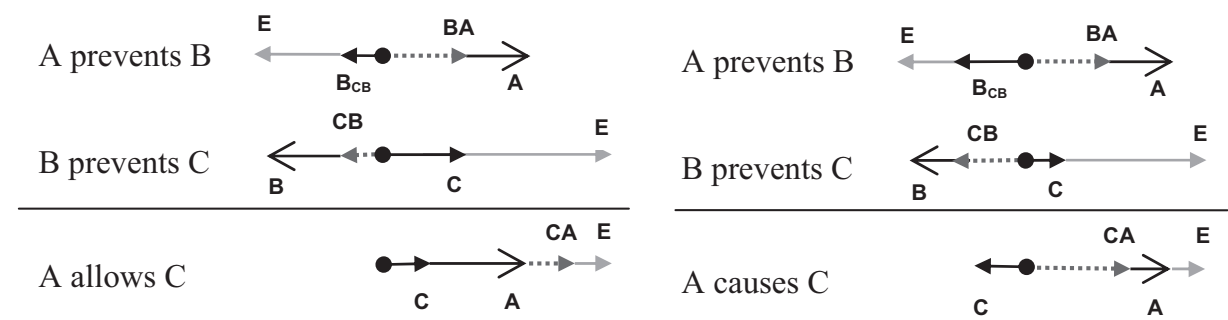

Figure 7. The composition of two PREVENT relations can lead to either an ALLOW (left side) or a CAUSE (right side) conclusion.

$100 \%$ of the time. ${ }^{2}$ Thus, according to the force theory, A causes $\neg B$ is virtually synonymous with $A$ prevents $B$.

\section{Testing the Force Theory}

In summary, the force theory makes the following predictions: (a) Causation by omission is understood in terms of the second and third entities in a double prevention. (b) The relation between the first and third entity in a double prevention chain is either an ALLOW or a CAUSE relation, depending on the relative strength of the forces associated with the second and third entities in the chain. (c) In the absence of clear knowledge of the magnitudes, double preventions will be most naturally described as ALLOW relations. (d) As discussed later in the present article, if a double prevention is followed by a CAUSE relation, then the most likely conclusion will be a CAUSE relation.

We tested these first four predictions in Experiments 1 and 2. In these experiments, participants viewed animations instantiating configurations of forces associated with various causal relations, including double preventions. The key question was which causal expressions people would choose in order to describe the chains.

The fifth prediction made by the force theory concerns causal chains beginning with PREVENT relations. In the force theory and other theories of causation, PREVENT relations involve counterfactual reasoning: They are about events that would have occurred had they not been kept from happening. Because these events do not occur, certain patterns of forces are not realized. Although such patterns might not occur, they can nevertheless be predicted. We refer to such anticipated but unrealized forces as virtual forces. In Experiment 3, we examined how such forces influence the way a causal chain is described.

A sixth set of predictions made by the force theory concerns various equivalence relationships between different expressions of causation. For example, as already noted, the force theory predicts that the expression $A$ causes $\neg B$ is virtually synonymous with $A$ prevents $B$. This is only one of several correspondences predicted by the force theory. As it turns out, dependency theories also predict various correspondences between expressions of causation (see Appendix B). These correspondence relations offer a way by which the predictions of the force theory and various dependency theories can be directly compared. Such comparisons are made in Experiment 4 by having people select which expressions are closest in meaning to other expressions. In summary, the assumptions of the force theory can be tested by examining how people choose to describe animations of causal events as well as how they relate various types of causal expressions.

\section{Experiment 1}

In Experiment 1, participants were shown five different animations, each depicting three cars, labeled $A, B$, and $C$, interacting with one another by pushing or pulling with a rope. The animations instantiated five types of chains: a CAUSE/CAUSE chain, a CAUSE/PREVENT chain, and three kinds of PREVENT/ PREVENT chains (see Figure 8). The CAUSE/CAUSE and CAUSE/PREVENT chains were included in order to provide examples of chains that, according to the theory, should not be described in terms of causation by omission. Of central interest was how people would describe the three types of PREVENT/ PREVENT chains. The directions and speeds of the cars were calculated using a physics simulator based on forces generated from a computer implementation of the force theory. Participants saw each animation four times. For each animation, they were presented with one of four different lists of four possible descriptions from which they were to select the most appropriate one. The first list included descriptions concerning the relationship between A and $\mathrm{C}$, that is, (a) A caused $C$ to cross the line, (b) A allowed $C$ to cross the line, (c) A prevented $C$ from crossing the line, and (d) None of the sentences above are applicable to the scene. Option (d) was the same in all lists. The second list of options described the causer as absent, that is, (a) The absence of A's influence caused $C$ to cross the line, (b) The absence of A's influence allowed $C$ to cross the line, (c) The absence of A's influence prevented $C$ from crossing the line, and (d) None of the sentences above are applicable to the scene. The other two lists of options were the same as the first two except that the descriptions concerned the relationship between $\mathrm{B}$ and $\mathrm{C}$.

In the CAUSE/CAUSE animation, $\mathrm{A}$ hits $\mathrm{B}, \mathrm{B}$ hits $\mathrm{C}$, and $\mathrm{C}$ crosses the line. The predictions of the force theory for this causal chain as well as for the other causal chains are shown in Table 3. Specifically, the theory predicts that people should be willing to say $A$ caused $C$ to cross the line and $B$ caused $C$ to cross the line, but not that the absence of A or B had any effect on $\mathrm{C}$. Thus, when presented with a list of sentences describing the effects of absences, people should choose the None of the above option. For the CAUSE/PREVENT chain, A hits B while $\mathrm{C}$ approaches the line from the opposite direction, and then $\mathrm{B}$ hits $\mathrm{C}$ and stops it from

\footnotetext{
${ }^{2}$ When the magnitude of the patient is 0 , the configuration of forces is potentially compatible with $A$ causes $\neg B$ but not $A$ prevents $B$, and it is because of this one difference that the two expressions are not perfectly synonymous.
} 

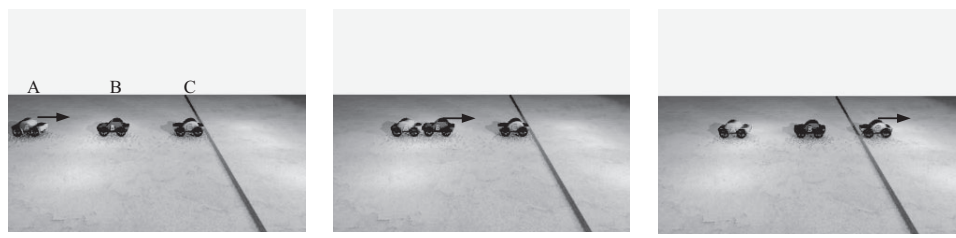

CAUSE/CAUSE (A/B caused C to cross the line.)
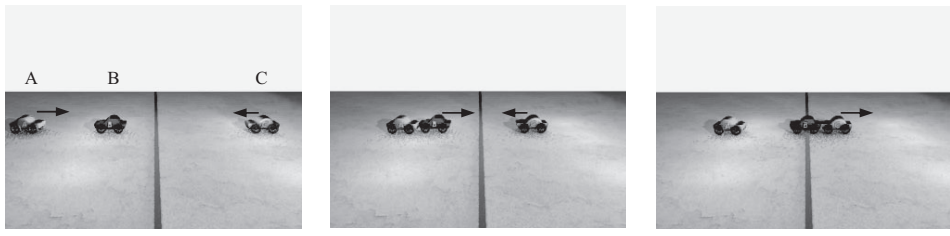

CAUSE/PREVENT (A/B prevented C from crossing the line.)
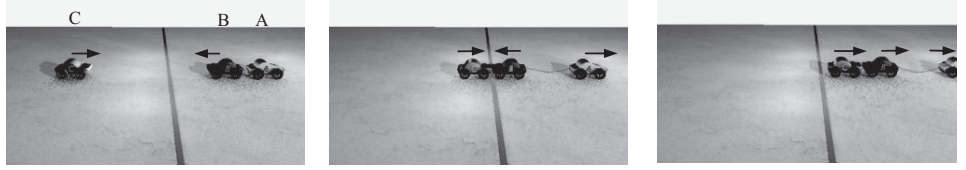

PREVENT/ PREVENT-1 (A allowed C to cross the line.; Lack of B allowed C to cross the line.)
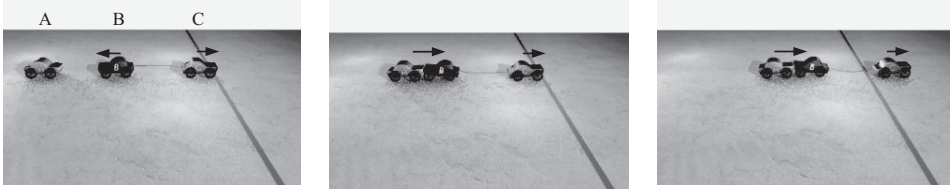

PREVENT/ PREVENT-2 (A allowed C to cross the line.; Lack of B allowed C to cross the line.)
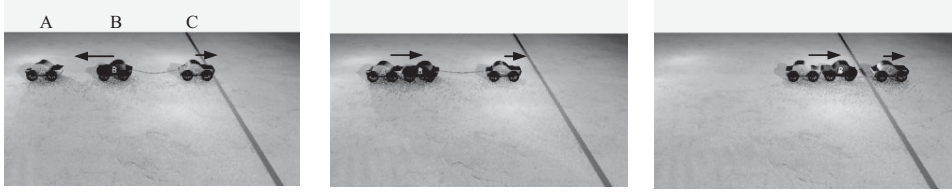

PREVENT / PREVENT-3 (A/B caused/allowed $C$ to cross the line.; Lack of B allowed $C$ to cross the line.)

Figure 8. Frames depicting the beginning, middle, and ending of animations used in Experiment 1, which instantiated CAUSE/CAUSE, CAUSE/PREVENT, and three types of PREVENT/PREVENT, along with some of the causal expressions used to describe them.

crossing the line. The force theory predicts that people should be willing to say $A$ prevented $C$ from crossing the line and $B$ prevented $C$ from crossing the line, and nothing else. When presented with lists of sentences describing the effects of absences, people should choose the None of the above option. The three PREVENT/ PREVENT causal chains implemented different ways in which double prevention can be physically realized. We included the three types of double preventions to establish the generalizability of the force theory's predictions as well as to test the prediction that the appropriateness of either a CAUSE or an ALLOW description depends on the magnitude of the patient forces in the double prevention.

In one of these double preventions (see Figures 4 and 8), P/P-1, $\mathrm{C}$ approaches the line, but is prevented from doing so by $\mathrm{B}$, which pushes against $\mathrm{C}$; $\mathrm{A}$ then pulls $\mathrm{B}$ away via a rope, preventing the prevention, and $\mathrm{C}$ proceeds to cross the line. When $\mathrm{B}$ and $\mathrm{C}$ meet, they came to a standstill, clearly indicating that their forces are the same. According to the force theory, when the B and C forces are the same, the conclusion is unambiguously ALLOW. As a consequence, it was predicted that people would be willing to say $A$ allowed $C$ to cross the line. Less clear was whether participants would be willing to say $B$ prevented $C$ from crossing the line, because the prevention occurred during only the first half of the animations. When none of the expressions stood out as best, we predicted that people would choose the None of the above option. The key prediction for the P/P-1 animation, as well as for the other double prevention animations, was that people would be willing to describe the relationship between $\mathrm{B}$ and $\mathrm{C}$ in terms of causation by omission, specifically, The lack of B's influence allowed $C$ to cross the line.

The second P/P-2 animation showed another instance of double prevention. In this animation, $\mathrm{C}$ approaches the line, but is pre- 
Table 3

Predicted Modal Responses and Observed Mean Proportions of Responses for Five Types of Chains With Associated Standard Errors (SEs)

\begin{tabular}{|c|c|c|c|c|c|}
\hline Response & CAUSE/CAUSE & CAUSE/PREVENT & $\mathrm{P} / \mathrm{P}-1$ & $\mathrm{P} / \mathrm{P}-2$ & $\mathrm{P} / \mathrm{P}-3$ \\
\hline$A$ caused $C$ & $.90(.056)^{\mathrm{a}}$ & - & $.10(.056)$ & $.27(.082)$ & $.43(.092)^{\mathrm{a}}$ \\
\hline$A$ allowed $C$ & $.07(.046)$ & - & $.73^{*}(.082)$ & $.70^{*}(.085)$ & $.47^{*}(.093)$ \\
\hline A prevented $C$ & - & $.90(.056)^{\mathrm{a}}$ & - & - & - \\
\hline None of the above & $.03(.033)$ & $.10(.056)$ & $.17(.069)$ & $.03(.033)$ & $.10(.056)$ \\
\hline$B$ caused $C$ & $.63(.089)^{a}$ & - & $.07(.046)$ & $.23(.079)$ & $.07(.046)$ \\
\hline$B$ allowed $C$ & $.27(.082)$ & - & $.27(.082)$ & $.13(.063)$ & $.17(.069)$ \\
\hline$B$ prevented $C$ & - & $.87(.063)^{\mathrm{a}}$ & $.17(.069)$ & $.23(.079)$ & $.13(.063)$ \\
\hline None of the above & $.10(.056)$ & $.13(.063)$ & $.50(.093)^{a}$ & $.40(.091)^{\mathrm{a}}$ & $.63(.089)^{a}$ \\
\hline Lack of A caused $C$ & $.03(.033)$ & - & - & - & $.07(.046)$ \\
\hline Lack of A allowed $C$ & $.07(.046)$ & $.07(.046)$ & $.13(.063)$ & $.07(.046)$ & $.07(.046)$ \\
\hline Lack of A prevented $C$ & $.03(.033)$ & $.13(.063)$ & $.13(.063)$ & $.17(.069)$ & $.17(.069)$ \\
\hline None of the above & $.87(.063)^{a}$ & $.80(.074)^{a}$ & $.73(.082)^{\mathrm{a}}$ & $.77(.079)^{a}$ & $.70(.085)^{\mathrm{a}}$ \\
\hline Lack of B caused $C$ & $.23(.079)$ & $.03(.033)$ & $.07(046)$ & $.10(.056)$ & $.07(.046)^{\mathrm{a}}$ \\
\hline Lack of $B$ allowed $C$ & $.13(.063)$ & $.10(.056)$ & $.50(.093)^{\mathrm{a}}$ & $.53(.093)^{\mathrm{a}}$ & $.50(.093)^{\mathrm{a}}$ \\
\hline Lack of $B$ prevented $C$ & $.10(.056)$ & $.23(.079)$ & - & $.07(.046)$ & $.03(.033)$ \\
\hline None of the above & $.53(.093)^{\mathrm{a}}$ & $.63(.089)^{a}$ & $.43(.092)$ & $.30(.085)$ & $.40(.091)$ \\
\hline
\end{tabular}

Note. $\quad \mathrm{P} / \mathrm{P}=\mathrm{PREVENT/PREVENT.} \mathrm{Boldface} \mathrm{type} \mathrm{indicates} \mathrm{observed} \mathrm{modal} \mathrm{response.} \mathrm{Dashes} \mathrm{indicate} \mathrm{the} \mathrm{absence} \mathrm{of} \mathrm{any} \mathrm{responses.}$

${ }^{\text {a }}$ Predicted modal response.

vented from crossing it by $\mathrm{B}$, which pulls $\mathrm{C}$ back via a rope; $\mathrm{A}$ then pushes against $\mathrm{B}$, preventing its prevention, and $\mathrm{C}$ proceeds to cross the line. The kinematics in the $\mathrm{P} / \mathrm{P}-1$ and $\mathrm{P} / \mathrm{P}-2$ animations differed: Whereas in $\mathrm{P} / \mathrm{P}-1 \mathrm{~B}$ pushed against $\mathrm{C}$, in $\mathrm{P} / \mathrm{P}-2$, $\mathrm{B}$ pulled $\mathrm{C}$, and whereas in $\mathrm{P} / \mathrm{P}-1$ A pulled $\mathrm{B}$, in $\mathrm{P} / \mathrm{P}-2 \mathrm{~A}$ pushed $\mathrm{B}$. Although the kinematics in the animations differed, the underlying forces were the same, and so the predictions for these animations are the same as well, as indicated in Table 3.

As discussed previously, the force theory predicts that double preventions can lead to both ALLOW and CAUSE relations between the first and last entities in the chain, depending on the magnitude of the forces. In the first two animations, the magnitudes of the forces were such that people were predicted to strongly prefer to characterize the relationship between $\mathrm{A}$ and $\mathrm{C}$ as an ALLOW relation rather than as a CAUSE relation (e.g., $A$ allowed $C$ to cross the line). In P/P-3, the magnitudes of the $\mathrm{B}$ and $\mathrm{C}$ forces were changed so that, according to the force theory, they should lead to CAUSE rather than to ALLOW responses. As in $\mathrm{P} / \mathrm{P}-2$, in $\mathrm{P} / \mathrm{P}-3, \mathrm{C}$ approached the line, but was prevented from crossing it by $\mathrm{B}$, which pulled it back via a rope, until $\mathrm{A}$ pushed against $\mathrm{B}$, preventing its prevention, and $\mathrm{C}$ was then able to cross the line. It should be noted that whereas animations can clearly show when two underlying forces are the same (e.g., by showing $\mathrm{B}$ and $\mathrm{C}$ at a standstill), it is very difficult for animations to depict the relative strength of two forces when the two forces act on different objects. As shown in Figure 7, a double prevention implies a CAUSE relation when the magnitude of the force associated with $\mathrm{B}$ is twice that of $\mathrm{C}$. Thus, whereas the underlying forces used to create the $\mathrm{P} / \mathrm{P}-3$ animation were those predicted to give rise to a CAUSE conclusion between $\mathrm{A}$ and $\mathrm{C}$, we expected that people might sometimes choose ALLOW descriptions due to uncertainty about the exact difference in magnitudes of the forces. Once again, one of the key predictions for the P/P-3 animation was that people would be willing to describe the relationship between $\mathrm{B}$ and $\mathrm{C}$ as instantiating causation by omission. Given the magnitude of the forces, we predicted that people would be willing to endorse the CAUSE statement, The lack of B's influence caused $C$ to cross the line. However, because of uncertainty about the magnitude of the forces, people might also endorse the ALLOW statement, The lack of B's influence allowed $C$ to cross the line.

\section{Predictions of Dependency Theories}

In addition to the predictions of the force theory, several predictions can be drawn for dependency theories. Strictly speaking, the probabilistic contrast model cannot make predictions for how people will describe the animations shown in this experiment because each animation is shown only once, and a single observation is not enough to establish the conditional probabilities needed for attributing causation or prevention. Assuming people could establish these probabilities on the basis of background knowledge (Lien \& Cheng, 2000), the probabilistic contrast model would say that the CAUSE/CAUSE and the CAUSE/PREVENT chains lead to different overall conclusions (CAUSE and PREVENT, respectively), but it could not say that the CAUSE/ CAUSE chain was associated with a different conclusion than the PREVENT/PREVENT chains (which should lead to ALLOW and CAUSE conclusions), because the statistical properties of the two chains are the same: In both types of chains, the presence of the cause raises the probability of the effect. ${ }^{3}$ Nor could counterfactual theories determine that the CAUSE/CAUSE and PREVENT/ PREVENT chains lead to different conclusions because such theories do not distinguish CAUSE from ALLOW.

The mental model theory is able to distinguish the CAUSE/ CAUSE chain from the double prevention chains. According to the mental model theory, in ALLOW relations, the affector is neces-

\footnotetext{
${ }^{3}$ Double preventions can lead to both CAUSE and ALLOW conclusions. In both types of conclusions, the presence of the initial entity raises the probability of the effect, that is, $\mathrm{P}($ Effect $\mid$ Initial entity) $>\mathrm{P}($ Effect $\neg$ Initial entity).
} 
sary for the effect, whereas in CAUSE relations the affector is sufficient for the effect (Goldvarg \& Johnson-Laird, 2001; Wolff, 2007). In the animations used in this experiment, the initial entity in the CAUSE/CAUSE chain can be viewed as sufficient for the effect, whereas the initial entity of the PREVENT/PREVENT chains can be viewed as necessary for the effect. However, the mental model theory would not be able to predict different descriptions for the various PREVENT/PREVENT chains, specifically, that P/P-3 might be more associated with a CAUSE conclusion than either $\mathrm{P} / \mathrm{P}-1$ or $\mathrm{P} / \mathrm{P}-2$, as predicted by the force theory. With respect to the causal model theory, it is not clear how this theory would distinguish the CAUSE/CAUSE chain from PREVENT/PREVENT chains. According to the causal model theory, ALLOW relations are more complex than CAUSE relations because they require an accessory variable (see Appendix B). However, as specified in Table A1, the number of forces used to create the CAUSE/CAUSE and PREVENT/PREVENT chains were the same. Assuming that the causal model theory could distinguish the CAUSE/CAUSE and PREVENT/PREVENT chains, it would not be able to distinguish different types of double prevention. Furthermore, none of the theories predict that causation by omission should be associated with double prevention chains. Rather, in all of these theories, causation by omission is viewed as simply an absence or a nonoccurrence of an event or a causal factor, and it is for this reason that they are all vulnerable to the causal selection problem, as discussed earlier.

\section{Method}

Participants. The participants were 30 Emory University undergraduates who took part in the study for course credit. All participants were native speakers of English.

Materials. Five animations were made from an animation package called 3ds Max 8 (Autodesk, Inc., 2006). The animations instantiated the following chains: CAUSE/CAUSE, CAUSE/ PREVENT, PREVENT/PREVENT-1 (P/P-1), PREVENT/ PREVENT-2 (P/P-2), and PREVENT/PREVENT-3 (P/P-3). Frames depicting the beginning, middle, and end of the animations are shown in Figure 8. The direction and speed of the cars was calculated by a physics simulator called Havok Reactor, a subprogram of 3ds Max. The mass of each car in this simulated world was $5 \mathrm{~kg}$ (approximately 11 pounds). Table A1 shows the magnitude and direction of the forces in newtons entered into the physics simulator for each car in each animation. Each animation depicted three cars labeled $A, B$, and $C$. In every animation, A was green, $\mathrm{B}$ was red, and $\mathrm{C}$ was blue. The cars moved over a gray cement surface, and the sky was a slightly lighter gray.

As a manipulation check, a separate group of Emory University undergraduates $(N=20)$ indicated whether the animations could be described as instantiating double prevention. Participants were shown the five animations described above and asked whether the animation could be described with the sentence $A$ prevented $B$ from preventing $C$ from crossing the line. (The other option was The sentence above is not applicable to the scene above.) As predicted, for the CAUSE/CAUSE and CAUSE/PREVENT animations, participants chose the double prevention description only $3 \%$ and $17 \%$ of the time, respectively. In contrast, for the three PREVENT/PREVENT animations, the double prevention description was chosen $97 \%$ of the time for $\mathrm{P} / \mathrm{P}-1,73 \%$ of the time for
$\mathrm{P} / \mathrm{P}-2$, and $80 \%$ of the time for $\mathrm{P} / \mathrm{P}-3$. The results from the preliminary rating task confirmed that the PREVENT/PREVENT animations were likely to be viewed as intended, that is, as instantiating double preventions.

Procedure and design. The animations were presented on Windows-based computers using E-Prime (Version 2.0) by Psychology Software tools (2008). Participants were told that they would see a series of animations in which cars bumped into or pulled one another. Below each animation were listed four possible descriptions; participants were to select the one that best described the animation. Each animation was presented four times. For two of these times, the possible descriptions named the first and last cars in the causal chain (A and C); for the other two times, the possible expressions named the second and last cars in the causal chain (B and C). Within both sets of descriptions, half described the causer as present, e.g., $A \_C$ to [from] cross[ing] the line with the blank filled in with caused, allowed, or prevented. The other half of the sentences described the causer as absent, for example, The absence of A's influence $C$ to $[$ from] cross[ing] the line. All the sets of options included None of the sentences above are applicable to the scene above as the fourth option. Participants were instructed to choose the sentence that best described what actually occurred in the scene, not what could have occurred. Participants were allowed to replay the animations as many times as they wanted before indicating their answers by clicking a radio button next to their choice. The animations were presented in a different random order for each participant.

\section{Results and Discussion}

The results provide the first empirical demonstration that causation by omission can be grounded in physical forces, and further that people are willing to use CAUSE and ALLOW verbs even when force is not transmitted from the initial to the final entity in a causal chain. As can be seen in Table 3, in cases in which only a single response was predicted, the force theory predicted the most frequent response 15 out of the 15 times. In the two cases in which two responses were predicted (see P/P-3 column in Table 3), the force theory correctly predicted one but not the other set of responses. In the first case, it correctly predicted that $A$ caused $C$ to cross the line and $A$ allowed $C$ to cross the line would be chosen in similar proportions. In the other case, the force theory also predicted that both the ALLOW and the CAUSE sentences would be chosen when describing $\mathrm{B}$ as absent; however, contra our predictions, participants strongly preferred The lack of $B$ allowed $C$ over The lack of $B$ caused $C$. We discuss this finding in greater detail in the following sections.

With respect to the CAUSE/CAUSE chain, participants chose the sentence containing cause to describe the relationship between $\mathrm{A}$ and $\mathrm{C}, \chi^{2}(2, N=30)=43.4, p<.0001$, as well as between $\mathrm{B}$ and $\mathrm{C}, \chi^{2}(2, N=30)=13.4, p=.0001$. As predicted, participants preferred None of the above when describing the relationships in terms of the absence of $\mathrm{A}, \chi^{2}(3, N=30)=60.9, p<.0001$, and in terms of the absence of $\mathrm{B}, \chi^{2}(3, N=30)=14, p=.0003$.

In the case of the CAUSE/PREVENT chain, participants chose the sentence containing prevent to describe the relationship between $\mathrm{A}$ and $\mathrm{C}, \chi^{2}(1, N=30)=19.2, p<.0001$, as well as between $\mathrm{B}$ and $\mathrm{C}, \chi^{2}(1, N=30)=16.13, p<.0001$. Also as predicted, participants preferred None of the above to describe the 
relationship in terms of the absence of $\mathrm{A}, \chi^{2}(2, N=30)=29.6$, $p<.0001$, and in terms of the absence of $\mathrm{B}, \chi^{2}(3, N=30)=26$, $p<.0001$.

As expected for the P/P-1 animation, participants chose the sentence containing allow to describe the relationship between A and $\mathrm{C}$ more often than the other options, $\chi^{2}(1, N=24)=13.5$, $p<.001$. With respect to the relationship between $\mathrm{B}$ and $\mathrm{C}$, responses were nonrandom, the modal response being None of the above, $\chi^{2}(3, N=30)=12.4, p=.006$. This result is not surprising given that the force theory is compatible with several possible relationships. As predicted, participants were not willing to endorse any of the statements beginning with The absence of A's influence preferring instead None of the above, $\chi^{2}(2, N=30)=$ 21.6, $p<.0001$. Most importantly, for statements beginning with The absence of B's influence, the overall pattern of responses differed from chance, $\chi^{2}(2, N=30)=9.80, p=.007$, with the modal response being The absence of $B$ 's influence allowed $C$ to cross the line, though this expression did not differ from None of the above, $\chi^{2}(1, N=28)=0.143, p=.705$.

The responses to the $\mathrm{P} / \mathrm{P}-2$ animation were much the same as to the $\mathrm{P} / \mathrm{P}-1$ animation. Participants chose the sentence containing allow to describe the relationship between $\mathrm{A}$ and $\mathrm{C}$ more often than the other options, $\chi^{2}(1, N=29)=5.828, p=.016$. With respect to the relationship between $\mathrm{B}$ and $\mathrm{C}$, the modal response was None of the above, but it did not differ significantly from chance, $\chi^{2}(3, N=30)=4.4, p=.221$. As predicted, participants did not endorse any of the statements beginning with The absence of $A$ 's influence, $\chi^{2}(2, N=30)=25.8, p<.0001$, choosing instead None of the above. Critically, as for statements beginning with The absence of B's influence, the overall pattern of responses differed from chance, $\chi^{2}(3, N=30)=13.2, p=.004$, with the modal response being The absence of $B$ 's influence allowed $C$ to cross the line. For these statements, there was a relatively high number of None of the above responses. However, if absent cause and absent allow responses are combined, they differ significantly from None of the above responses, $\chi^{2}(1, N=28)=5.14, p=$ .023 , offering direct support for the hypothesis that double preventions can be interpreted in terms of absences. It is worth emphasizing that although the arrangement of the cars in the P/P-2 animation differed from that in the P/P-1 animation (cf. rows 3 and 4 in Figure 8), the underlying forces in both animations were the same (see Table A1). Thus, the similar responses to the P/P-1 and P/P-2 animations indicate that the results were dependent not on a particular kinematic pattern of motion, but rather on the underlying configuration of forces.

In the case of the P/P-3 animation, the force magnitudes were such that if they were perceived accurately, then they should give rise to a preponderance of CAUSE descriptions for the relationship between $\mathrm{A}$ and $\mathrm{C}$. However, as discussed previously, because the exact magnitude of these forces cannot be determined by sight, it was expected that people might also choose ALLOW descriptions. As it turned out, there was no preference to choose either CAUSE or ALLOW expressions over the other, $\chi^{2}(1, N=27)=0.037$, $p=.847$, a result that contrasts with what we found for the P/P-1 and $\mathrm{P} / \mathrm{P}-2$ animations. The higher level of CAUSE responses in the case of the P/P-3 animation supports the prediction of the force theory that the choice between CAUSE and ALLOW in double preventions depends on the magnitude of the forces. With respect to the relationship between $\mathrm{B}$ and $\mathrm{C}$, participants' responses were nonrandom, the modal response being None of the above, $\chi^{2}(3$, $N=30)=24.13, p<.0001$. This result is not surprising because the force theory is compatible with several possible relationships in this case. With respect to statements in terms of absences, participants chose None of the above, $\chi^{2}(3, N=30)=33.2, p<$ .0001 , for statements about the absence of A's influence. Interestingly, participants' preferences for statements beginning with The absence of $B$ 's influence did not differ from chance, $\chi^{2}(1, N=$ $27)=333, p=.564$. The predicted modal response for this type of expression was a CAUSE expression. We found, in contrast, that people appeared to prefer ALLOW expressions, which is in contrast to their responses to the relationship between A and C, which split roughly evenly between cause and allow responses.

In summary, the results provide support for several of the key predictions of the force theory. First, they show that people are willing to describe a causal chain with CAUSE and ALLOW relations even when a force is not transmitted from the initial cause to the effect. Second, the results support the hypothesis that double preventions can be re-expressed in terms of omissions. Third, the results show that double preventions and, by extension, omissions can be instantiated in terms of configurations of force. As discussed earlier, some theorists have held that process theories are incapable of specifying the occurrence of effects from omission (Menzies, 2006; Schaffer, 2000; Schulz et al., 2007; Woodward, 2006). The present findings demonstrate that this criticism is incorrect. Fourth, the results support the perhaps surprising prediction that the difference between double preventions associated with CAUSE and ALLOW depends, in part, on the relative magnitude of the patient forces.

The results cannot be readily explained by dependency theories. In contrast to the probabilistic contrast model and counterfactual theories, people gave different conclusions to CAUSE/CAUSE chains than double prevention chains. In contrast to the predictions of the mental model and the causal model theories, CAUSE responses were used more frequently to describe the P/P-3 animation than the P/P-1 and P/P-2 animations. Furthermore, none of the theories predict that causation by omission should be associated with double preventions.

Although the results supported the force theory, there were two findings that are not readily explained by it. First, in the case of the P/P-3 animation, the relationship between the initial and final entities divided roughly evenly between CAUSE and ALLOW expressions, whereas the underlying forces were those that should have led to CAUSE conclusions. As we noted earlier, people may have sometimes chosen ALLOW expressions rather than CAUSE expressions because they were uncertain about the relative magnitudes of the patient forces and because double preventions more often result in ALLOW than CAUSE conclusions, according to the force theory. In addition, the lower than expected number of CAUSE responses might have to do with the way the animations unfolded over time. Intuitively, double preventions implying CAUSE relationships tend to involve chains in which the causing and resulting parts of the chain occur together in time. Consider, for example, a situation in which a person opens a closet door and boxes tumble out. People would probably prefer to say that opening the door caused rather than allowed the boxes to fall out. In this kind of situation, the causing and resulting events occur virtually simultaneously. In contrast, in the animations used in Experiment 1, the two parts of the event were teased apart. As a consequence, 
people may have focused more heavily on the last part of the event, the car crossing the line, which would lead to a preponderance of ALLOW descriptions, because the last car to move had a tendency for the endstate.

This account might help explain another curious finding in the data. In the case of the P/P-3 animation, people used both CAUSE and ALLOW expressions to describe the relationship between A and $\mathrm{C}$, but to describe the relationship between the lack of $\mathrm{B}$ and $\mathrm{C}$, people strongly preferred ALLOW over CAUSE expressions. The expression The lack of B's influence may have served to focus people's attention even more exclusively on the last part of the animation, because the expression lack of B's influence treats the force associated with $B$ as part of a cause rather than an effect of the casual chain. If only the actions of the last car are considered, then the only reasonable expressions would be The lack of $B$ 's influence allowed $C$ to cross the line, rather than The lack of B's influence caused $C$ to cross the line. In summary, the ways in which the data in Experiment 1 differed from what was predicted may have to do with how the events in the double preventions unfolded over time.

\section{Experiment 2}

In the previous experiment, when describing double preventions in terms of the lack of B's influence, participants preferred to use the verb allow over cause. It was noted that the infrequent use of CAUSE expressions may have been due to the temporal characteristics of the events. In order for double preventions to give rise to CAUSE conclusions, it may sometimes be necessary for the double prevention chain to be followed by a CAUSE relation. Consider, for example, a scenario in which a person drops a rock into a pool of water. The situation involves a double prevention: A person prevents his or her grip on the rock, which leads to its falling. The situation can be aptly described by the expression The absence of the person's grip allowed the rock to fall into the pool. However, if a CAUSE relation is added to the end of this double prevention, then the preferred interpretation may shift toward a CAUSE relation. For example, if we add the causal relation the rock's falling into the pool caused the water to ripple, it sounds more natural to describe the entire chain as The absence of the person's grip on the stone caused the water to ripple than The absence of the person's grip allowed the water to ripple.

These intuitions are consistent with the force theory, which predicts that when a CAUSE relation is added to a double prevention, the patient force in the overall conclusion will be biased away from the endstate, making CAUSE conclusions more frequent than ALLOW conclusions (Barbey \& Wolff, 2007, 2009). The theory predicts, then, that people should be more willing to say that lack of $B$ caused $D$ given the chain A PREVENTS B, B PREVENTS C, $C$ CAUSES D, than to say that lack of $B$ caused $C$ given the chain A PREVENTS B, B PREVENTS C.

We developed three new animations to examine this prediction. Each of these animations started with double preventions involving a reflexive relationship in which an entity prevented its own prevention. A reflexive double prevention occurs when, for example, a person releases a pencil, and the pencil falls to the ground. Initially, the person prevents the falling, and then the person prevents her own prevention by releasing her grip. In terms of cars, a reflexive double prevention was implemented by having a car release a rope that was preventing another car from moving. Instantiating double preventions in terms of releasing simplifies the causal chain and makes it easier to add relations.

The three new animations are depicted in Figure 9. The first row depicts a simple releasing event that we refer to as ALLOW-byreleasing. In this animation, B moves toward the line but is prevented from crossing it by a rope attached to A. After a few moments, A releases the rope, and B crosses the line. To keep the animations as simple as possible, "releasing" was depicted by the rope's becoming detached from the car. The process could have been interpreted as the rope breaking, but if so, the resulting event would still be an instance of double prevention, and so the predictions would be the same. The forces associated with this double prevention are shown in Figure 10. At first, A's external influence on $\mathrm{B}$ prevents it from crossing the line. Next, a force that is internal to A (e.g., a decision or an internal motor motion) prevents A's external influence on B by releasing the rope. The theory predicts that the animation should be described with the expression The absence of A's influence allowed B to cross the line.

CAUSE-by-Releasing, depicted in the second row of Figure 9, includes a third car, $\mathrm{C}$, to represent an additional CAUSE relation. The causal chain begins the same way as ALLOW-by-Releasing, with A releasing $\mathrm{B}$, but then $\mathrm{B}$ pushes $\mathrm{C}$ across the line. As noted earlier, a double prevention followed by a CAUSE relation should lead to a causation-by-omission conclusion. Specifically, the force theory predicts that a double prevention followed by a CAUSE relation should lead to $\neg \mathrm{A}$ cause $\mathrm{C}$ conclusions $49 \%$ of the time and $\neg \mathrm{A}$ allows $\mathrm{C}$ conclusions $41 \%$ of the time. ${ }^{4}$

The animation in the third row in Figure 9 exemplifies a double prevention followed by a PREVENT relation, or PREVENT-byReleasing. Again, the causal chain begins with ALLOW-byReleasing. In this case, however, the release of $\mathrm{B}$ prevents $\mathrm{C}$ from crossing the line. This animation was predicted to give rise to $\neg A$ prevents $C$ responses.

The key issue addressed in Experiment 2 was whether adding a causal relation to a double prevention would result in more causing-by-omission interpretations than allowing-by-omission interpretations, as predicted by the force theory. The animations included all of the animations used in Experiment 1, plus the animations depicted in Figure 9. As in Experiment 1, participants watched the animations and then indicated which of several possible expressions best described the animation. Unlike in Experiment 1 , each animation was followed by only one list of nine descriptions that was held constant across all the animations. The nine options on the list included cause, allow, and prevent descriptions expressed in terms of the presence of the initial car in the chain and in terms of its absence. In the interest of completeness, the list also included statements in which the result was negated, for example, A caused $C$ to not cross the line (though we did not expect such statements to be the modal response for any of the animations). Given that the number of possible expressions was relatively high, their order was systematically varied across three conditions to offset any potential order effects.

For the animations shown in Experiment 1, we predicted that we would replicate the findings from that experiment. For the new

\footnotetext{
${ }^{4}$ The remaining $10 \%$ of the conclusions should be associated with None of the above responses.
} 


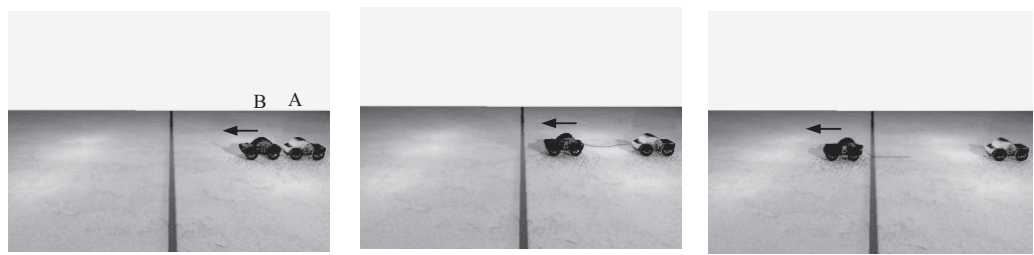

ALLOW-by-Releasing (The absence of A's influence allowed B to cross the line.)
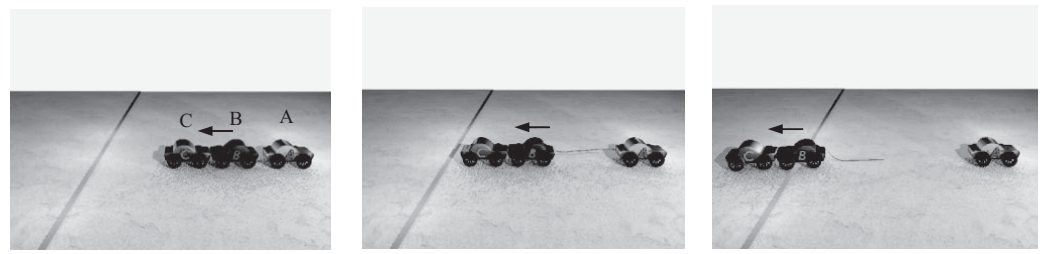

CAUSE-by-Releasing (The absence of A's influence caused C to cross the line.)
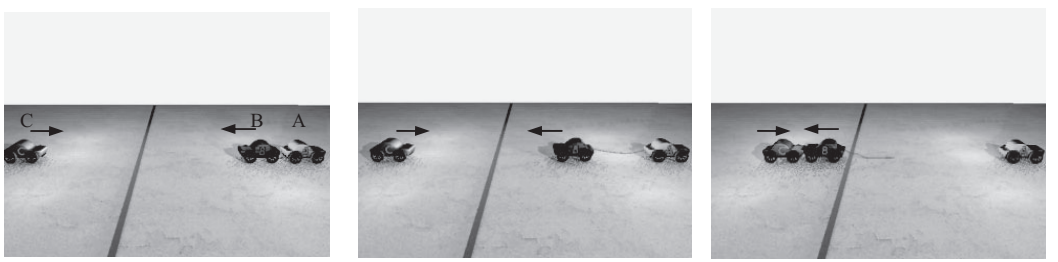

PREVENT-by-Releasing (The absence of A's influence prevented C from crossing the line.)

Figure 9. Frames depicting the beginning, middle, and ending of three animations used in Experiment 2, instantiating ALLOW-by-Releasing, CAUSE-by-Releasing, and PREVENT-by-Releasing, along with the causal expressions predicted to best describe them.

animations depicting releasing, we predicted that people would describe the ALLOW-by-Releasing animation with the sentence The absence of A's influence allowed $C$ to cross the line. For the CAUSE-by-Releasing animation, we predicted that people would choose The absence of A's influence caused $C$ to cross the line, more often than The absence of A's influence allowed $C$ to cross the line. Finally, for the PREVENT-by-Releasing animation, we predicted that people would prefer The absence of A's influence prevented $C$ from crossing the line.

\section{Method}

Participants. The participants were 75 Emory University undergraduates who took part in the study for course credit. All participants were native speakers of English.

$$
\mathrm{A}_{\text {Internal }} \text { prevents } \mathrm{A}_{\text {External }}
$$

A External prevents B

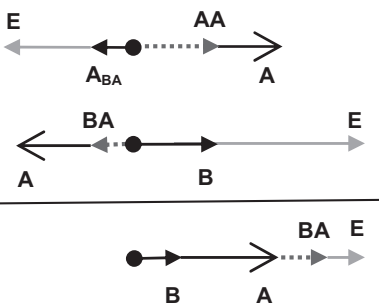

Figure 10. The configuration of forces associated with ALLOW-byReleasing. Initially, A's external influence on B prevents B from occurring, but then a force internal to A prevents A's external influence on B. The resulting composition of forces supports the expressions $A$ allowed $B$ and $\neg$ A allows $B$.
Materials. The materials included the five animations used in Experiment 1, specifically, CAUSE/CAUSE, CAUSE/ PREVENT, P/P-1, P/P-2, and P/P-3. Three additional animations, Release-CAUSE, Release-ALLOW, and ReleasePREVENT, were constructed in the same way as those in Experiment 1. As shown in Figure 9, each of the new animations depicted either two or three cars, labeled with letters, moving over a gray cement surface. Table A2 shows the magnitudes and directions of the forces in newtons entered into the physics simulator for each car used in the three new animations for this experiment.

Procedure and design. The animations were presented on Windows-based computers using E-Prime (Version 2.0) by Psychology Software tools (2008). Participants were told that they would see a series of animations in which cars bumped into or pulled one another. Below each animation, participants read nine expressions, specifically: A caused $C$ to cross the line; $A$ allowed $C$ to cross the line; A prevented $C$ from crossing the line; The absence of A's influence caused $C$ to cross the line; The absence of A's influence allowed $C$ to cross the line; The absence of A's influence prevented $C$ from crossing the line; $A$ caused $C$ to not cross the line; A allowed $C$ to not cross the line; $A$ prevented $C$ from not crossing the line. In addition, the list included a 10th option, None of the sentences above are applicable to the scene above. The 75 participants were evenly divided into three groups, each of which saw the list of options in a different order. The first group saw the sentences in the order shown above. The list for the second group had the first 
three options moved to the end of the list. Similarly, the third list had the first three options from List 2 moved to the bottom. Participants were instructed to choose the sentence that best described what actually occurred in the scene, not what could have occurred, by clicking a radio button next to their choice. Participants could replay the animations as many times as they wanted before making a choice. The animations were presented in a different random order for each participant.

\section{Results and Discussion}

The results supported the predictions of the force theory. Table 4 shows the proportion of times people endorsed various possible descriptions for the eight animations. In terms of modal responses, the force theory predicted the most frequent response in 7 out of 7 times a single response was predicted. For the one animation in which two modal responses were predicted, $\mathrm{P} / \mathrm{P}-1$, participants chose the two modal responses roughly equally.

The results replicated the findings from Experiment 1 . Specifically, for the CAUSE/CAUSE chain, the modal response was $A$ caused $C, \chi^{2}(5, N=75)=265, p<.0001$. For the CAUSE/ PREVENT chain, the preferred sentence was A prevented $C, \chi^{2}(5$, $N=75)=88.1, p<.0001$. For the P/P-1 and P/P-2 animations, the modal response was allow, $\chi^{2}(5, N=75)=132.3, p<.0001$; $\chi^{2}(6, N=75)=200.4, p<.0001$. In contrast, for the P/P-3 animation, "cause" and "allow" sentences were selected nearly equally, $\chi^{2}(7, N=75)=141.9, p<.0001$.

The three new animations, CAUSE-by-Releasing, ALLOWby-Releasing, and PREVENT-by-Releasing, allowed us to examine double prevention followed by another relation. The predictions regarding the new animations were also borne out. The ALLOW-by-Releasing animation was most often described with the statement The absence of A's influence allowed $C$ to cross the line, $\chi^{2}(4, N=75)=123.9, p<.0001$. The overall pattern of responses to the CAUSE-by-Releasing animation differed from chance, $\chi^{2}(7, N=75)=114.1, p<.0001$, with the modal response being The absence of $A$ 's influence caused $C$ to cross the line, followed by The absence of A's influence allowed $C$ to cross the line. The number of absent CAUSE responses did not differ from the number of absent ALLOW responses, $\chi^{2}(1, N=58)=0.276, p=.599$. However, the number of absent CAUSE responses to this animation was greater than the number of absent CAUSE responses to the ALLOW-by-Releasing animation, $\chi^{2}(1, N=42)=9.5, p=$ .002. Furthermore, the observed proportion of CAUSE and ALLOW responses for the CAUSE-by-Releasing animation, .41 and .36 , were very close to the percentages predicted by the force theory, .47 and .43 . The evidence supports, then, the hypothesis that adding a causal relation to a double prevention shifts responses from allowing-by-omission to causation-byomission. For the PREVENT-by-Releasing animation, the modal response was The absence of A's influence prevented $C$ from crossing the line, $\chi^{2}(5, N=75)=140.6, p<.0001$.

In summary, the results provide further evidence that double preventions can give rise to ALLOW or CAUSE relations. The key finding in this experiment is that when a double prevention is followed by an additional CAUSE relation, the causal chain can be described as causing an absence. The results provide further support for the hypothesis that causation by omission can be explained in terms of a process model. They also provide further evidence against dependency theories. Most importantly, certain chains involving double preventions led to CAUSE conclusions and others to ALLOW conclusions. As described earlier, none of the dependency theories are able to explain how this might come about. In addition, none of the theories predict that causation by omission is associated with double preventions.

\section{Experiment 3}

At first glance, it might seem that chains beginning with PREVENT relations should not be possible. For example, if A first prevents $B$, and then $B$ prevents $C$, the second prevent should not be able to occur because $\mathrm{B}$ has been prevented. However, as shown in Experiments 1 and 2, one way in which such chains can be realized is if the order of the relations is reversed, that is, if $\mathrm{B}$ first prevents $\mathrm{C}$, and then $\mathrm{A}$ prevents $\mathrm{B}$. As it turns out, there may be another way in which double preventions can be realized. The motivation for this second approach begins with the observation that PREVENT relations are about events that would have occurred had they not been

Table 4

Predicted Modal Responses and Observed Proportions of Responses for Eight Types of Chains Used in Experiment 2 With Associated Standard Errors (SES)

\begin{tabular}{|c|c|c|c|c|c|c|c|c|}
\hline Response & $\mathrm{C} / \mathrm{C}$ & $\mathrm{C} / \mathrm{P}$ & $\mathrm{P} / \mathrm{P}-1$ & $\mathrm{P} / \mathrm{P}-2$ & $\mathrm{P} / \mathrm{P}-3$ & $\begin{array}{l}\text { CAUSE-by- } \\
\text { Releasing }\end{array}$ & $\begin{array}{l}\text { ALLOW-by- } \\
\text { Releasing }\end{array}$ & $\begin{array}{c}\text { PREVENT-by- } \\
\text { Releasing }\end{array}$ \\
\hline A caused $C$ & $.86(.040)^{a}$ & $.01(.013)$ & $.12(.038)$ & $.25(.051)$ & $.39(.057)^{\mathrm{a}}$ & - & - & - \\
\hline$A$ allowed $C$ & $.05(.026)$ & - & $.71(.053)^{a}$ & $.63(.056)^{a}$ & $.45(.057)^{\mathrm{a}}$ & $.04(.023)$ & $.05(.026)$ & $.01(.013)$ \\
\hline$A$ prevented $C$ & - & $.52(.058)^{\mathrm{a}}$ & - & - & $.01(.013)$ & $.03(.019)$ & $.01(.013)$ & $.08(.032)$ \\
\hline Absence of $A$ caused $C$ & - & $.03(.019)$ & $.01(.013)$ & - & - & $.41(.057)^{\mathrm{a}}$ & $.15(.041)$ & - \\
\hline Absence of $A$ allowed $C$ & - & - & $.04(.022)$ & - & $.03(.019)$ & $.36(.056)$ & $.71(.053)^{\mathrm{a}}$ & - \\
\hline Absence of $A$ prevented $C$ & $.01(.013)$ & - & $.01(.013)$ & $.03(.019)$ & $.01(.013)$ & $.03(.019)$ & - & $.67(.055)^{\mathrm{a}}$ \\
\hline A caused lack of $C$ & $.03(.019)$ & $.28(.052)$ & - & $.03(.019)$ & - & $.01(.103)$ & $.01(.103)$ & $.08(.031)$ \\
\hline$A$ allowed lack of $C$ & - & - & $.01(.013)$ & $.03(.019)$ & $.03(.019)$ & - & - & - \\
\hline A prevented lack of $C$ & $.01(.013)$ & $.08(.031)$ & - & - & $.01(.013)$ & $.01(.103)$ & - & $.01(.103)$ \\
\hline None of the above & $.03(.019)$ & $.08(.032)$ & $.09(.034)$ & $.04(.022)$ & $.07(.029)$ & $.11(.036)$ & $.07(.029)$ & $.15(.041)$ \\
\hline
\end{tabular}

Note. $\quad \mathrm{C} / \mathrm{C}=\mathrm{CAUSE} / \mathrm{CAUSE} ; \mathrm{C} / \mathrm{P}=\mathrm{CAUSE} / \mathrm{PREVENT} ; \mathrm{P} / \mathrm{P}=\mathrm{PREVENT} / \mathrm{PREVENT}$. Boldface type indicates observed modal response.

a Predicted modal response. 
kept from occurring (Dowe, 2000; Walsh \& Sloman, 2005, 2009). On a psychological level, recognizing a PREVENT relation involves counterfactual reasoning because it requires envisioning what would have occurred in the absence of the blocking event. This raises a possibility: If A prevents B and B prevents $\mathrm{C}$, it may not be necessarily that the second prevent in the chain-B prevents $\mathrm{C}$-actually occur; all that might be needed is that the second prevention be anticipated.

The early parts of a causal chain may sometimes make it possible to anticipate later parts of the causal chain. For example, it is well known that people spontaneously extrapolate the movement of moving objects to positions beyond their current location, a phenomenon known as representational momentum (Freyd \& Finke, 1985; Hubbard, 1995). On the basis of this process, people may be able to infer the future realization of events, and their associated forces, before they actually occur. We refer to such anticipated but unrealized forces as virtual forces. Such forces may allow people to anticipate and represent the configurations of forces following a PREVENT relation such that they do not actually have to be physically realized in order for them to be considered as components of a causal chain and entered into the process of relation composition.

The idea that virtual forces might enter into people's representations of causal chains can be illustrated with pairs of closely related animations, one in which all of the forces are actual and the other in which one of the forces is virtual. Consider, for example, the simple interaction of forces depicted in Figure 11. In this animation, B moves toward the line; A then moves toward the line and hits B, preventing it from crossing. The forces associated with this animation are shown above the sequence of still frames.

A slight modification of this sequence of events is shown in Figure 12. The animation presented in Figure 12 begins the same way as in the one shown in Figure 11: B approaches the line, and then A approaches B. However, in Figure 12, A stops several feet before reaching the line, and $\mathrm{B}$ crosses the line. If $\mathrm{A}$ had not stopped, then it would have prevented B from crossing the line, but some force (internal to A) prevented this interaction from occurring.
The sequence of events shown in Figure 12 can be viewed as instantiating a double prevention, as shown in the free-body diagrams in Figure 12. A's movement toward B implies that A will prevent $\mathrm{B}$. This anticipated event constitutes the second prevent relation in the double prevention. However, something inside of $\mathrm{A}$ prevents it from continuing. This internal process brings about the first PREVENT relation. Composing these two relations leads to an ALLOW relation, specifically, that $A$ allowed $B$ to cross the line or The absence of A's influence allowed $B$ to cross the line. Thus, people might treat virtual forces as if they were actual forces.

According to the force theory, virtual relations and forces can only be realized in causal chains beginning with PREVENT relations. Importantly, the relation following the initial PREVENT relation need not be another PREVENT relation. It is possible that virtual forces may apply in chains such as A prevents B and B causes C, as investigated by Walsh and Sloman (2005; see also 2009):

There is a bottle on the wall. Frank and Jane are standing close by. While they are there someone else aims to throw a ball at the bottle. The aim is perfectly on target. Frank and Jane both step in front of the bottle. Frank happens to step in front of Jane and catches the ball. The bottle doesn't break (p. 2332)

Walsh and Sloman (2005) found that participants were willing to say that Frank, but not Jane, prevented the bottle from breaking. People's willingness to form a link between Frank and the bottle implies that they represented the situation in the form of a causal chain, specifically, Frank prevents ball and ball causes bottle breaking. The second relation in this causal chain-ball causes bottle breaking-was never realized. Nevertheless, people's willingness to form a relationship between Frank and the bottle breaking suggests that people were willing to factor this virtual relation into their causal judgments. They may have been willing to factor in the nonoccurring CAUSE relation because the motion of the ball was such that it implied that the CAUSE relation would have occurred had Frank not intervened. Thus, people's interpretation of the situation appeared to involve the composition of a

\section{$\mathrm{A}_{\text {External }}$ prevents B}
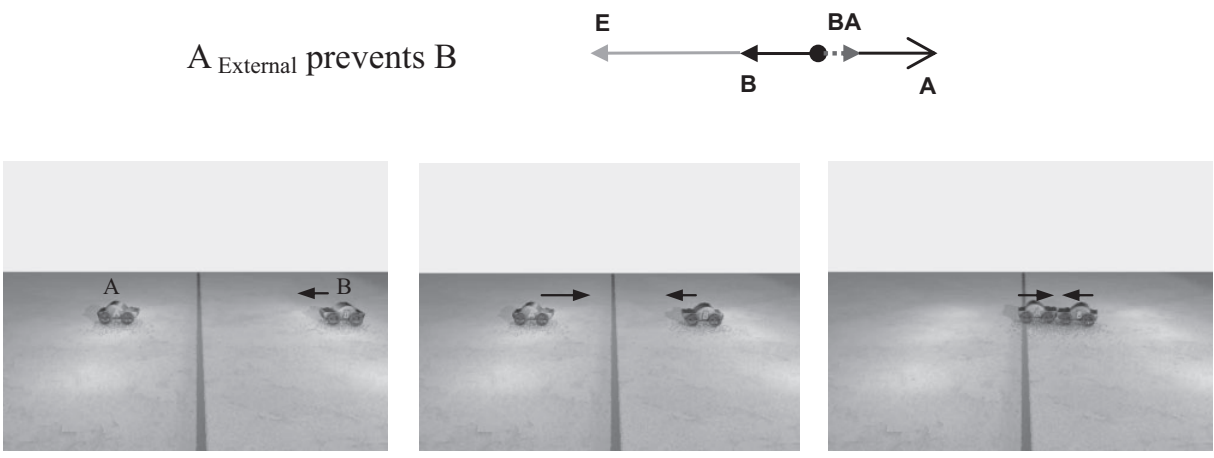

PREVENT

Figure 11. The sequence of events instantiates a PREVENT relation. B approaches the line, then A approaches $\mathrm{B}$ and prevents it from crossing the line. The sequence of events can be described with the sentence A prevented $B$ from crossing the line. The configuration of forces associated with this sequence of events is shown above the still frames. 


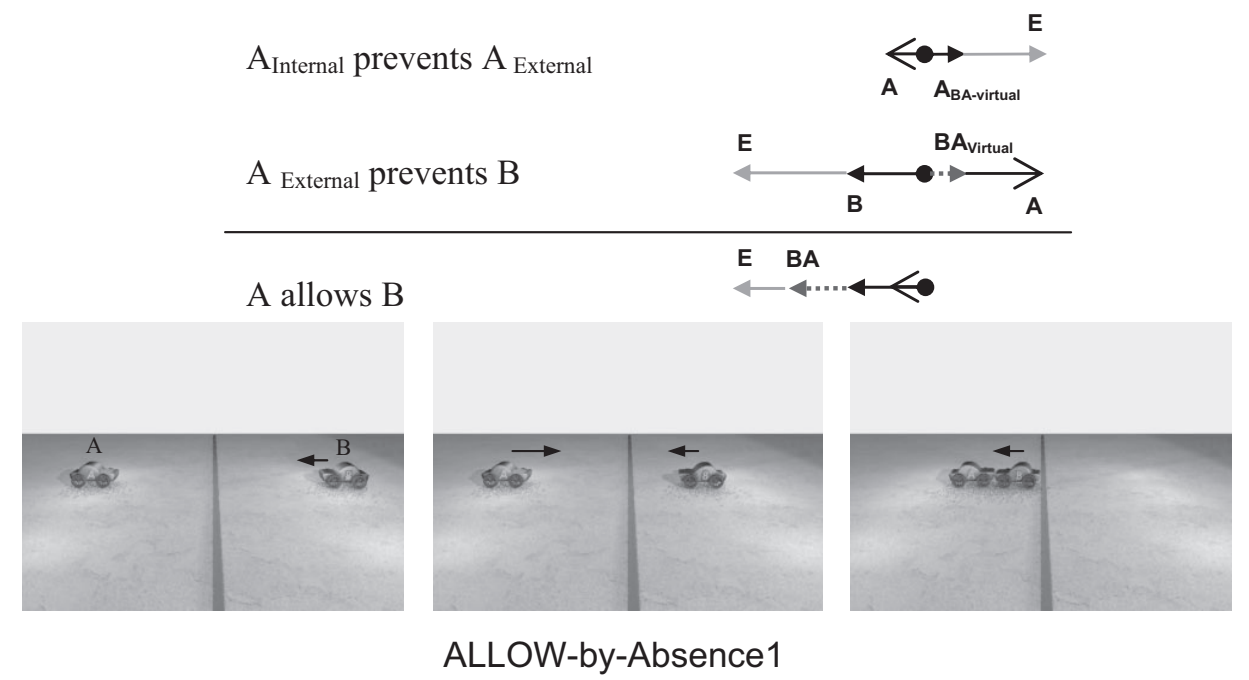

Figure 12. The sequence of events instantiates allowing-by-omission. B approaches the line, then A approaches $\mathrm{B}$, threatening to prevent it from crossing the line; at the last moment, however, A stops and B crosses the line. The sequence of events can be interpreted as The absence of A's influence allowed $B$ to cross the line. The configuration of forces associated with this sequence of events is shown above the still frames.

PREVENT and a (virtual) CAUSE relation, leading to a PREVENT relation, just as Walsh and Sloman (2005) observed. ${ }^{5}$

In Experiment 3, we investigated the idea that relation composition can be based on both actual and virtual forces. Specifically, we examined whether causal chains involving virtual forces could lead to relation compositions resulting in the conclusions $A$ prevented $B, A$ allowed $C$, and The absence of $A$ allowed $C$. In addition to the pair of animations in Figures 11 and 12, we constructed four other pairs of animations to examine the extent to which people base their descriptions on virtual forces.

One of these pairs is shown in Figure 13. The still frames shown on the top row come from an animation used in Experiments 1 and 2, CAUSE/PREVENT, in which C moves toward the line, and then A moves toward and hits $\mathrm{B}$, which then hits $\mathrm{C}$, preventing $\mathrm{C}$ from crossing the line. In Experiments 1 and 2, people indicated that this animation could be described by the expression $A$ prevented $C$ from crossing the line. The animation depicted below, ALLOWby-Absence 2 , is very similar to the CAUSE/PREVENT animation, except that it involves virtual forces. As in CAUSE/PREVENT, the ALLOW-by-Absence 2 animation begins with $\mathrm{C}$ moving toward the line while A moves toward B, threatening to hit it. However, at the last moment, A comes to a stop, leaving B where it is, and $\mathrm{C}$ is able to cross the line. We propose that A's stopping itself is a PREVENT relation, and hence the animation as a whole instantiates a PREVENT/CAUSE/PREVENT chain, which, according to the force theory, implies an ALLOW conclusion (Barbey \& Wolff, 2009). Hence, the force theory predicts that people should be willing to describe the animation with the sentence The absence of $A$ allowed $C$ to cross the line. Note that in this PREVENT/CAUSE/PREVENT chain, the first PREVENT relation is realized, whereas the following CAUSE and PREVENT relations are inferred.

A third pair of animations is shown in Figure 14. The animation shown on the top row was used in Experiment 2. In it, $\mathrm{C}$ moves toward the line, and then $\mathrm{B}$, which is tethered to A, moves toward
$\mathrm{C}$ to block it. After a brief struggle, A releases B, which then ultimately prevents $\mathrm{C}$ from crossing the line. In Experiment 2, this animation was described with the sentence The absence of A's influence prevented $C$ from crossing the line. The animation depicted in the second row of Figure 14 is the same as the one in the first row, except that A does not release B. Even though B is not released, it appears that if $\mathrm{B}$ had been released, it would have prevented $\mathrm{C}$ from crossing the line. We propose that this strong impression of a threatened PREVENT relation will lead people to imagine what would have happened if $\mathrm{B}$ had been released and interpret the causal chain as if the PREVENT was actually realized. In other words, we expect that this chain will be viewed as a double prevention - one actual and one virtual, and hence should give rise to an ALLOW conclusion, specifically, $A$ allowed $C$ to cross the line.

\footnotetext{
${ }^{5}$ Walsh and Sloman (2005) investigated a scenario in which a spinning coin would have landed on tails had it been hit by a rolling ball, but the ball was intercepted by a person (Frank), and the coin landed on heads. People were unwilling to ascribe causation to Frank. Walsh and Sloman interpreted this finding as consistent with mechanistic theories that require a chain of physical interactions. Although we agree with Walsh and Sloman's overall conclusion, we think the results may have occurred for different reasons. In our view, this scenario instantiates a double prevention-Frank prevents ball and ball prevents tails-in which the second prevent relation involves virtual forces. People resisted ascribing causation to this scenario not because the mechanism was incomplete, but rather because double preventions are more often better described with ALLOW expressions. We suspect that if given the chance, people would be willing to endorse the statement "Frank's intervention allowed the ball to land on heads." The availability of an ALLOW option might also have implications for research showing that people's attributions of causation are affected by the intentionality of the causer and causee in a causal scenario (Wolff, 2003), especially in the case of double preventions (see Lombrozo, 2009).
} 

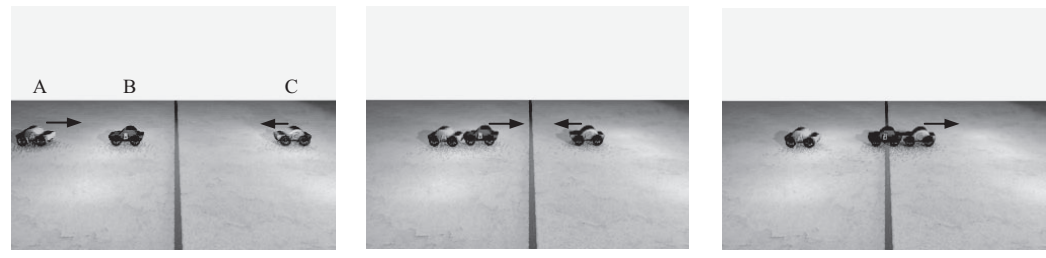

CAUSE/PREVENT (A prevented C from crossing the line.)
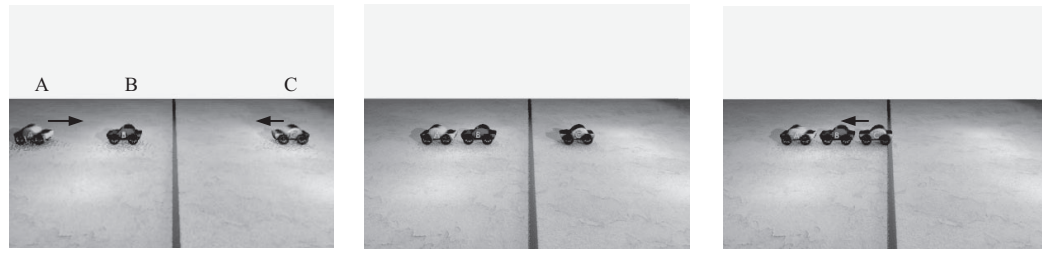

ALLOW-by-Absence2 (The absence of $A$ 's influence allowed $C$ to cross the line.)

Figure 13. The animation in the first row depicts causing a prevention: $\mathrm{C}$ approaches the line while A approaches $\mathrm{B}$, hitting $\mathrm{B}$ into $\mathrm{C}$ and preventing $\mathrm{C}$ from crossing the line. The animation can be describe with the expression A prevented $C$ from crossing the line. The animation in the second row is the same as in the first row, except that A stops before hitting $\mathrm{B}$, and as a consequence, $\mathrm{B}$ does not run into $\mathrm{C}$ and $\mathrm{C}$ crosses the line. This animation can be described with the expression The absence of A's influence allowed $C$ to cross the line.

The fourth pair of animations is shown in Figure 15. The still frames on the top row come from an animation used in Experiment 2 in which $\mathrm{B}$ moves toward $\mathrm{C}$ and is briefly prevented from hitting $\mathrm{C}$ by a tether to $\mathrm{A}$; then $\mathrm{A}$ releases $\mathrm{B}$, and $\mathrm{B}$ pushes $\mathrm{C}$ over the line. People indicated that this animation was well described by the expression The absence of A's influence caused C to cross the line. In this animation, all of the forces were actual, whereas in the animation in the second row, some of the forces were virtual. The animation PREVENT-by-Holding begins like the animation CAUSE-by-Releasing, in that B moves toward C and is kept from hitting it by $\mathrm{A}$. Unlike in the previous animation, in this animation A continues to hang onto $\mathrm{B}$, so $\mathrm{C}$ remains where it is and does not cross the line. It was predicted that people would assume that if $\mathrm{B}$ were released, it would be able to push $\mathrm{C}$ over the line. On the basis of this virtual force, $\mathrm{C}$ had a tendency to cross the line, but A kept this tendency from being realized. As a consequence, it was
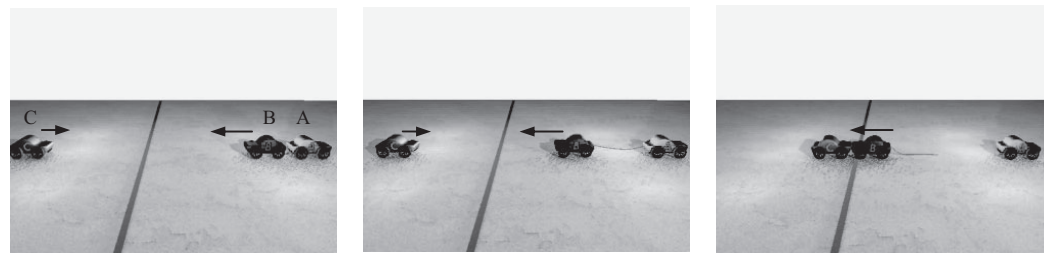

PREVENT-By-Releasing (The absence of A's influence prevented C from crossing the line.)
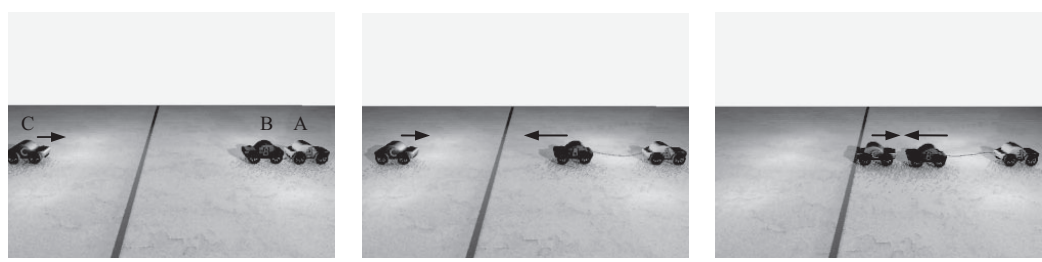

ALLOW-by-Holding (A allowed C to cross the line.)

Figure 14. The animation in the first row depicts prevention-by-omission: B and C both approach the line, from opposite directions; B is temporarily stopped because it is tethered to A; ultimately, however, A releases the rope and $\mathrm{B}$ reaches $\mathrm{C}$ in time to prevent it from crossing the line. This animation can be described with the expression The absence of A's influence prevented $C$ from crossing the line. The animation in the second row is the same as in the first row, except that $\mathrm{A}$ does not release $\mathrm{B}$, and as a consequence, $\mathrm{C}$ crosses the line. The animation in the second row can be described with the expression A allowed C to cross the line. 


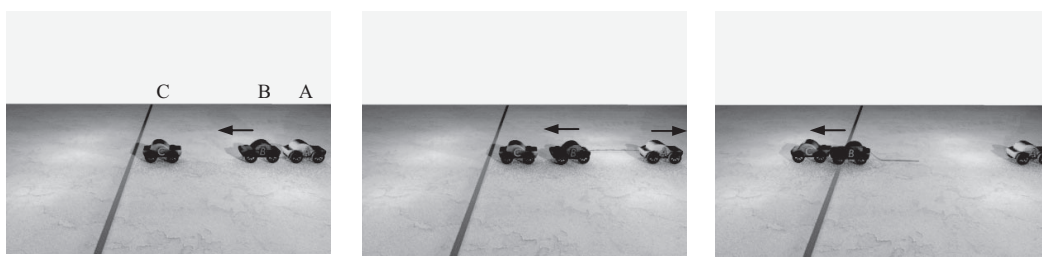

CAUSE-by-Releasing (The absence of $A$ 's influence caused $C$ to cross the line.)

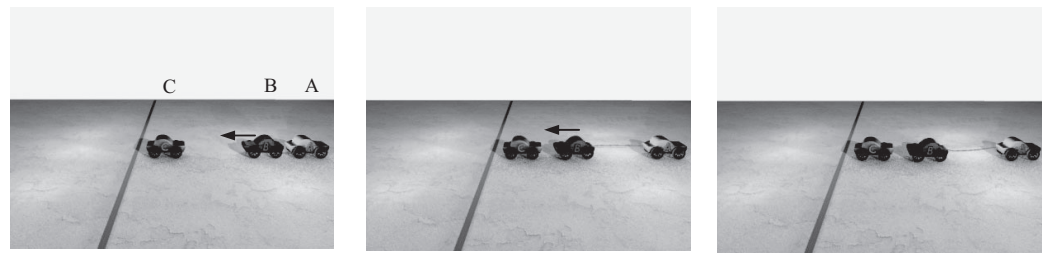

PREVENT-by-Holding (A prevented C from crossing the line.)

Figure 15. The animation in the first row depicts causing-by-releasing: B moves toward C, but is briefly held back from hitting $\mathrm{C}$ until A releases it, and then $\mathrm{B}$ causes $\mathrm{C}$ to cross the line. This animation can be described with the expression The absence of A's influence caused $C$ to cross the line. The animation in the second row is the same as in the first row, except that $\mathrm{A}$ does not release $\mathrm{B}$, and as a consequence, $\mathrm{C}$ does not cross the line. The animation in the second row can be described with the expression A prevented $C$ from cross the line.

predicted that people should be willing to describe the animation with the expression A prevented $C$ from crossing the line. One noteworthy aspect of this animation is that the cars remain essentially motionless throughout the animation, except for B's initial movement forward and a few small jiggles implying counteracting forces. The prediction is that, even without the occurrence of any significant movement, the mere anticipation of forces is enough to license a causal expression, under the assumption that people's descriptions factor in not only what occurs but also what would have occurred if other parts of the causal chain had not been present.

In the animations involving virtual forces, the participants could not know for sure whether an anticipated force was strong enough to bring about an effect because the effects were not shown but merely threatened. To help participants estimate the magnitude of the forces, we included both types of animations in the present experiment, shown in Figures 11-15. In the animations without virtual forces, participants could see what would occur if the anticipated forces in the remaining animations were, in fact, realized. As in previous experiments, participants watched animations of causal chains and then chose from a list of possible descriptions the one that best characterized what occurred. The experiment included a total of 10 animations: the four pairs of animations shown in Figures 11-15 plus the CAUSE/CAUSE and P/P-3 animations used in the previous experiments.

\section{Method}

Participants. The participants were 36 Emory University undergraduates who took part in the study for course credit. All participants were native speakers of English.

Materials. The materials included the five animations from Experiment 2: CAUSE/CAUSE, P/P-3, CAUSE/PREVENT, PREVENT-by-Releasing, and CAUSE-by-Releasing, and five new animations: namely, PREVENT (see Figure 11), ALLOWby-Absence1 (see Figure 12), ALLOW-by-Absence2 (see Figure
13), ALLOW-by-Holding (see Figure 14), and PREVENT-byHolding (see Figure 15). The new animations were constructed in the same way as in the previous experiments. Table A3 shows the magnitudes and directions of the forces in newtons entered into the physics simulator for each car in each of the five new animations used in this experiment.

Procedure and design. The procedure and design were the same as in Experiment 2. Participants viewed an animation and then chose one of nine possible expressions that best described the animation or the option None of the sentences above are applicable to the scene above.

\section{Results and Discussion}

The results supported the prediction that people would treat virtual forces as actual forces. Table 5 shows the proportion of times people endorsed various possible descriptions for the 10 animations. The force theory predicted the most frequent response for all 10 animations.

Responses to the animations used in previous experiments replicated previous results. Specifically, the CAUSE/CAUSE animation was most often described as A caused $C$ to cross the line, $\chi^{2}(2$, $N=36)=60.5, p<.0001$, and the P/P-3 animation was most often described as $A$ allowed $C$ to cross the line, $\chi^{2}(5, N=36)=$ 89.3, $p<.0001$.

Responses to the pairs of animations examining the effect of virtual forces were also as predicted. First, participants preferred to describe the PREVENT animation with the expression $A$ prevented $B$ from crossing the line, $\chi^{2}(2, N=36)=19.5, p<.0001$. The nearly synonymous expression $A$ caused $B$ not to cross the line was also often chosen. The corresponding animation involving virtual forces and labeled ALLOW-by-Absence1 was most often described with the expression The absence of A's influence allowed $B$ to cross the line, $\chi^{2}(2, N=36)=19.5, p<.0001$. We found the same pattern of results in the second pair of animations. For the CAUSE/PREVENT animation, participants most often 


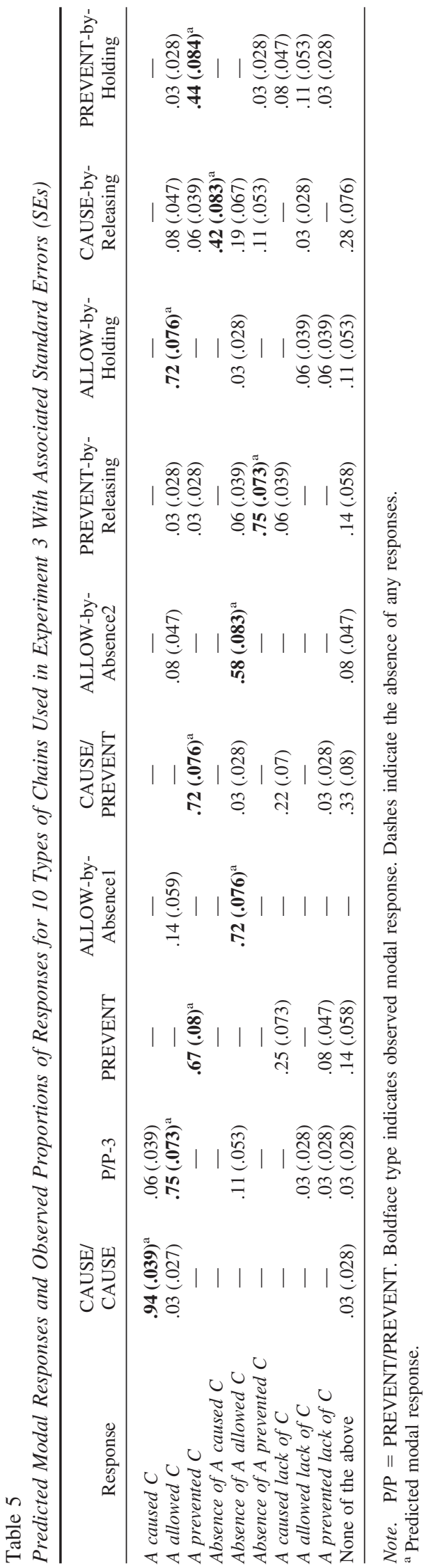

chose the expression $A$ prevented $C$ from crossing the line, $\chi^{2}(2$, $N=36)=19.5, p<.0001$. As with the PREVENT animation, a second popular choice was A caused B not to cross the line. For the corresponding animation depicting virtual forces, ALLOW-byAbsence2, people most often chose the expression The absence of $A$ 's influence allowed $C$ to cross the line, $\chi^{2}(2, N=36)=13.5$, $p<.0001$

The third pair of animations represented PREVENT-byReleasing and ALLOW-by-Holding. As in Experiment 2, participants' preferred expression for the PREVENT-by-Releasing animation was The absence of $A$ 's influence prevented $C$ from crossing the line, $\chi^{2}(5, N=36)=88.7, p<.0001$. Participants' preferred expression for the ALLOW-by-Holding animation, which involved virtual forces, was $A$ allowed $C$ to cross the line, $\chi^{2}(4, N=36)=62.6, p<.0001$. The final pair of animations represented CAUSE-by-Releasing and PREVENT-by-Holding. As in Experiment 2, the modal response to the CAUSE-by-Releasing animation was The absence of A's influence caused $C$ to cross the line, $\chi^{2}(6, N=36)=26.0, p<.0001$. The modal response to the animation involving virtual forces and labeled PREVENT-byHolding animation was $A$ prevented $C$ from crossing the line, $\chi^{2}(6$, $N=36)=36.7, p<.0001$. The response to PREVENT-byHolding is particularly noteworthy because, in effect, nothing happened in this animation, but the configuration of forces suggested what could have happened if certain forces had not been present, and such suggested forces were enough to license a PREVENT interpretation.

In this experiment, causal chains involving virtual forces were interpreted as if the forces were actual, as indicated by peoples' choice of causal expressions. Among other findings, virtual forces were especially effective in giving rise to statements about omissions, in particular, allowing by omission. These results provide further evidence that process theories are able to account for effects from omissions, while also avoiding the selection problem. As discussed earlier, the force theory is able to account for causation-by-omission because it does not require the transmission of energy from the initial to final entity in the causal chain. What the theory does require are the configurations of forces associated with different causal relations. There is no clear limit on how many of the forces can be virtual, but in general there need to be enough actual forces to allow for the anticipation of the virtual forces. The results from this experiment show that transmission of energy even between adjacent links in the chain is not always necessary. In certain circumstances, a force may be anticipated but never realized due to the unexpected intervention of another force. The results indicate that these suggested, but nonactualized forces may enter into how a causal chain is described.

\section{Experiment 4}

As discussed earlier, all theories of causation make predictions about how different kinds of causal expressions might be related to each other. Intuitively, certain causal expressions seem to have roughly the same meaning as other causal expressions. For example, the negative causation statement Lack of wind in the room allows the dust to settle seems to imply the positive causation statement Presence of wind in the room prevents the dust from settling. Likewise, Pain causes lack of sleep seems to have almost 
the same meaning as Pain prevents sleep. Other relationships are not so clear. When we say Aspirin prevents clotting, does this imply that Lack of aspirin causes clotting? Similarly, if Green tea prevents Alzheimer's, is it true that Lack of green tea causes Alzheimer's? Interestingly, nearly all of the dependency theories predict that these pairs of statements should have roughly the same meaning because they hold that $\neg$ A CAUSES B implies A PREVENTS B. The force theory also predicts this correspondence, but only weakly. To date, there have been no systematic examinations of the ways in which various types of causal expressions might be related to each other. This is somewhat surprising because nearly all theories of causation make predictions about how causal relations involving negation should be related to causal relations without negation.

The predictions made by each theory are derived in detail in Appendix B. For example, according to the probabilistic contrast model (Cheng \& Novick, 1992), causation is defined with respect to contrasting conditional probabilities. As explained in Appendix $\mathrm{B}$, the model implies correspondences between CAUSE $\neg$ and PREVENT, $\neg$ CAUSE and PREVENT, PREVENT $\neg$ and CAUSE, and $\neg$ PREVENT and CAUSE. Counterfactual theories define causation with respect to possible worlds. These theories imply a correspondence between CAUSE $\neg$ and PREVENT, PREVENT $\neg$ and CAUSE, and $\neg$ PREVENT and CAUSE. In Goldvarg and Johnson-Laird's (2001) mental model theory, causal relations are defined with respect to different sets of mental models. Their theory predicts correspondences between $\mathrm{CAUSE} \neg$ and PREVENT, CAUSE and PREVENT $\neg$, ALLOW and $\neg$ PREVENT, ALLOW $\neg$ and $\neg$ CAUSE, and $\neg$ ALLOW and PREVENT. According to Sloman et al.'s (2009) causal model theory, causal relationships are understood in terms of functional relationships that can be expressed in structural equations. The causal model theory predicts correspondences between CAUSE $\neg$ and PREVENT, ALLOW $\neg$ and PREVENT, PREVENT $\neg$ and ALLOW or CAUSE, $\neg$ CAUSE and PREVENT, $\neg$ ALLOW and PREVENT, $\neg$ PREVENT and ALLOW or CAUSE, CAUSE and $\neg$ PREVENT or PREVENT $\neg$, ALLOW and $\neg$ PREVENT or PREVENT $\neg$, and PREVENT and CAUSE $\neg$, $\neg$ ALLOW, $\neg$ CAUSE, or ALLOW $\neg$. The correspondences predicted by each of these dependency theories are summarized in Table 6 .

\section{Force Theory Approach to Causal Correspondences}

According to the force theory, the relationship between negative and positive causal statements can be explained in terms of subchains within an overall causal chain. For example, the statement Lack of $B$ allows $C$ implies a chain of two PREVENT relations, that is, A PREVENTS B and B PREVENTS C. If we want to know what would happen in the presence of $\mathrm{B}$, we eliminate the first premise and evaluate the chain with respect to the remaining premise. In this case, the remaining premise would be $B$ prevents $C$; hence, the theory predicts that the claim Lack of $B$ allows $C$ implies that Presence of $B$ prevents $C$. The strategy for deriving this implication can be generalized to other types of omission. For example, as shown in Experiments 2 and 3, the statement Lack of A causes $C$ implies a chain of two PREVENT relations followed by at least one CAUSE relation. To determine the relation implied when A is present rather than absent, we remove the initial PREVENT relation from the chain, resulting in the chain $B$ PREVENTS C, and C CAUSES D. For this subchain, the force theory predicts that $37 \%$ of the time the conclusion will be PREVENT, and $63 \%$ of the time the conclusion is undefined, meaning it does not have a simple expression in English. In other words, the theory predicts that lack of $A$ causes $C$ implies that the (presence of) $B$ prevents $C$ at a relatively modest level.

In all the statements considered so far, the chains involved absent causers. The force theory, as well as other theories, also makes predictions about how expressions describing absent effects are related to expressions in which the effect is present. For example, as discussed earlier, the expression related to A causes $\neg B$ can be determined by forming the causal chain A CAUSE $\mathrm{x}$, $x$ PREVENT B. Composing the relations in this chain leads to a PREVENT relation almost $100 \%$ of the time, hence the force theory predicts that $A$ causes $\neg B$ is virtually synonymous with $A$ prevents $B$. The same strategy can be applied to the expressions $A$

Table 6

The Predicted Proportion of Correspondences Associated With the Different Models, the Observed Proportions, and the Pearson Rs and RMSEs Between the Predicted and Observed Proportions From Experiment 4

\begin{tabular}{|c|c|c|c|c|c|c|}
\hline Response & $\begin{array}{l}\text { Probability- } \\
\text { raising models }\end{array}$ & $\begin{array}{l}\text { Counterfactual } \\
\text { models }\end{array}$ & $\begin{array}{l}\text { Mental model } \\
\text { theory }\end{array}$ & $\begin{array}{c}\text { Causal model } \\
\text { theory }\end{array}$ & $\begin{array}{l}\text { Force } \\
\text { theory }\end{array}$ & Observed \\
\hline$A$ caused B & $\neg \mathrm{P}(.5), \mathrm{P} \neg(.5)$ & $\neg \mathrm{P}(.5), \mathrm{P} \neg(.5)$ & $\mathrm{P} \neg(1)^{\mathrm{a}}$ & $\neg \mathrm{P}(.5), \mathrm{P} \neg(.5)$ & $\neg \mathrm{P}(.5), \mathrm{P} \neg(.5)$ & $\neg \mathrm{P}(.51), \mathrm{P} \neg(.24)$ \\
\hline$A$ allowed $B$ & - & - & $\neg \mathrm{P}(1)$ & $\neg \mathrm{P}(.5), \mathrm{P} \neg(.5)$ & $\neg \mathrm{P}(.5), \mathrm{P} \neg(.5)$ & $\neg \mathrm{P}(.63), \mathrm{P} \neg(.37)$ \\
\hline A prevented $B$ & $\mathrm{C} \neg(.5), \neg \mathrm{C}(.5)$ & $\mathrm{C} \neg(1)$ & $\mathrm{C} \neg(1)$ & $\begin{aligned} \mathrm{C} & \neg(.25), \neg \mathrm{A}(.25), \\
& \neg \mathrm{C}(.25), \mathrm{A} \neg(.25)\end{aligned}$ & $\begin{aligned} \mathrm{C} & \neg(.38), \neg \mathrm{A}(.38), \\
& \neg \mathrm{C}(.15), \mathrm{A} \neg(.08)\end{aligned}$ & $\begin{aligned} \mathrm{C} & \neg(.36), \neg \mathrm{A}(.34), \\
& \neg \mathrm{C}(.15), \mathrm{A} \neg(.05)\end{aligned}$ \\
\hline Absence of $A$ caused $B$ & $\mathrm{P}(1)$ & - & $\mathrm{A} \neg(1)^{\mathrm{a}}$ & $\mathrm{P}(1)$ & $\mathrm{P}(.38)$ & $\mathrm{P}(.62), \mathrm{A} \neg(.02)$ \\
\hline Absence of $A$ allowed $B$ & - & - & $\mathrm{P}(1)$ & $\mathrm{P}(1)$ & $\mathrm{P}(1)$ & $\mathrm{P}(.72)$ \\
\hline Absence of $A$ prevented $B$ & $\mathrm{C}(1)^{\mathrm{a}}$ & - & $\mathrm{A}(1)$ & $\mathrm{A}(.5), \mathrm{C}(.5)$ & $\mathrm{A}(.62), \mathrm{C}(.38)$ & $\mathrm{A}(.56), \mathrm{C}(.23)$ \\
\hline$A$ caused lack of $B$ & $\mathrm{P}(1)$ & $\mathrm{P}(1)$ & $\mathrm{P}(1)$ & $\mathrm{P}(1)$ & $\mathrm{P}(1)$ & $\mathrm{P}(.68)$ \\
\hline$A$ allowed lack of $B$ & - & - & $\neg \mathrm{C}(1)^{\mathrm{a}}$ & $\mathrm{P}(1)$ & $\mathrm{P}(.22)$ & $\mathrm{P}(.54), \neg \mathrm{C}(.02)$ \\
\hline A prevented lack of $B$ & $\mathrm{C}(1)^{\mathrm{a}}$ & $\mathrm{C}(1)^{\mathrm{a}}$ & $\mathrm{C}(1)^{\mathrm{a}}$ & $\mathrm{A}(.5), \mathrm{C}(.5)$ & $\mathrm{A}(.62), \mathrm{C}(.38)$ & $\mathrm{A}(.37), \mathrm{C}(.31)$ \\
\hline Pearson's $r$ & .55 & .46 & .57 & .91 & .95 & \\
\hline RMSE & .22 & .22 & .26 & .17 & .09 & \\
\hline
\end{tabular}

Note. $\mathrm{P}=$ PREVENT; $\mathrm{C}=\mathrm{CAUSE}$; $\mathrm{A}=\mathrm{ALLOW} ; \neg \mathrm{C}=$ for example, “ $\neg \mathrm{A}$ cause B"; $\mathrm{C} \neg=$ for example, "A cause $\neg \mathrm{B}$ "; $\mathrm{RMSE}=$ root-mean-square error between model and observed. Dashes indicate the absence of any responses.

${ }^{a}$ Missed prediction. 
allows $\neg B$. According to the force theory, the resulting relation composition results in PREVENT relations $24 \%$ of the time and undefined configurations $76 \%$ of the time. In other words, the force theory predicts that a statement such as A allows the absence of $C$ implies $A$ prevents $C$, but only weakly. Finally, according to the theory, A prevents $\neg B$ implies $A$ allows $C 62 \%$ of the time and A causes $C 38 \%$ of the time. All of the correspondence predictions made by the force theory are summarized in Table 6 . Also as shown in Table 6, the correspondences predicted by the force theory differ significantly from the other theories of causation.

In this experiment, we examined correspondence relationships between various causal expressions and how these relationships can be used to test different theories of causation. The procedure was simple: Participants read examples of various causal expressions and chose which sentence from a list of sentences was most similar in meaning to the standard sentence. The options varied with respect to the verb (cause, allow, prevent) and whether the cause or effect was expressed as present or absent. By studying the sentences participants chose, we could determine the relative similarity between the different types of causal expressions. These similarities were then compared against those predicted by the various theories.

\section{Method}

Participants. The participants were 48 Emory University undergraduates who took part in the study for course credit. All participants were native speakers of English.

Materials. The materials involved nine types of sentences: $A$ causes $B, A$ allows $B, A$ prevents $B, \neg A$ causes $B, \neg A$ allows $B, \neg A$ prevents $B$, A causes $\neg B$, A allows $\neg B$, A prevents $\neg B$. For each sentence type, we found 10 real-world examples using the Google and Yahoo search engines for a total of 90 causal sentences (see Table A4). ${ }^{6}$ Example sentences include Cell phones cause car accidents; Microscopes allow observation; Exercise prevents arthritis; Lack of light causes depression; Lack of drainage allows fluid accumulation; Lack of sleep prevents weight loss; Pain causes lack of sleep; Black holes allow no escape; Reservoirs prevent lack of water.

Procedure. Participants were run on Windows-based computers in sound-attenuating carrels using E-Prime (Version 2.0) by Psychology Software tools (2008). Participants were told that the experiment concerned how people understand the meaning of causal sentences. They were shown causal sentences one at a time. Each sentence was followed by a list of nine others (as listed above) as well as the option None of the above are related in meaning to the sentence above. The sentences in the list were altered to fit the content of the particular standard sentence. For example, for the sentence Exercise prevents arthritis, participants saw the sentences Exercise causes arthritis, Exercise allows arthritis, Exercise prevents arthritis, and so on. Participants were instructed to choose the sentence that was most similar to the sentence presented (but not the exact same sentence). The sentences were presented in random order on Windows-based computers.

Design. Participants were randomly assigned to one of two list versions. In each, participants made judgments on half of the materials, which included five examples of the nine types of causal sentences, for a total of 45 sentences for each participant. Within the two groups of participants, there were three subgroups. Each subgroup of three participants saw the list of options in a different order.

\section{Results and Discussion}

The results provided support for the force theory over the other theories. The first column in Table 6 shows the nine different types of causal expressions that were used as standards. Columns 2-6 show the types of causal relations that were predicted to correspond to these standards. The rightmost column shows the proportion of times that participants chose the predicted correspondences. The two bottom rows in Table 6 show the Pearson correlation and the root-mean-square error (RMSE) between the correspondences predicted by the models and those chosen by participants. RMSE is often used as a measure of accuracy or goodness of fit between the values predicted by a model and the values observed (Massaro, 2004). As shown in Table 6, the Pearson correlations and the RMSEs indicated a much stronger fit to the data for the force theory and the causal model theory than for the other theories.

Another way to analyze the data is in terms of modal responses. In counting the most frequent responses, we included those cases in which only one response was predicted, or in which only one response was predicted to dominate. As shown in Table 6, the force theory was able to predict the modal response in seven out of the nine cases, which was more than any other theory. The second best theory was the mental model theory, which was able to predict five out of the nine cases. Third best was the causal model theory, which was able to predict the modal response in four out of the nine cases. The models that were least able to predict people's modal responses were the probabilistic contrast and the counterfactual theories, which were able to account for only two of the nine cases.

The analyses so far provide the strongest support for the force theory, but also some support for the causal model and mental model theories. To distinguish between these three models, we used the Akaike information criterion (AIC). AIC values provide an estimate of information loss when the probability distribution associated with the true model is approximated by the probability distribution(s) implied by the model(s) to be tested (Wagenmaker $\&$ Farrell, 2004). The lower the AIC value, the more likely it is that the model approximates the model that was generated by the data (Ashby, 1992; Pitt \& Myung, 2002). Each model has an AIC value that is a function of the model's likelihood, $L$, and the number of parameters, $V(\mathrm{AIC}=-2 \log L+2 V)$. The mental model, causal

\footnotetext{
${ }^{6}$ Finding an example typically began with typing in a causal verb, sometimes with a negation, such as cause, allow, prevent, lack of causes, absence of causes, causes lack of, and then looking for results with an $A$-cause-verb-B structure. We sought examples in which the A and B terms consisted of only one or two words. Sometimes, one- or two-word arguments could not be found but could be created by adapting a longer phrase. For example, the sentence Erosion by rain and river that takes place in hilly areas causes landslides and floods could be simplified as Erosion causes landslides. In effect, the process of sampling the A and B arguments involved finding the "head" noun of the A and B term phrases, that is, the main noun that is modified by other elements in a noun phrase.
} 
model theory, and force theory can be construed as having one free parameter each. We used multinomial logistic regression to calculate the first term in the AIC formula, $-2 \log L$, by regressing the responses averaged over participants onto the responses predicted by the three models. The procedure indicated that the model with the lowest expected information loss was the force model (AIC = $100.08)$, followed by the causal model theory $(\mathrm{AIC}=105.91)$, and finally, by the mental model theory $(\mathrm{AIC}=106.76)$.

The AIC values can be made easier to interpret by transforming them into so-called Akaike weights (Wagenmaker \& Farrell, 2004). Akaike weights can be interpreted as the probability that a particular model is the best model given the data and the set of candidate models. The Akaike weight for the force model $\left(w_{F T}(\mathrm{AIC})=.92\right)$ was higher than that for both the causal model $\left(w_{C T}(\mathrm{AIC})=.05\right)$ and mental model theories $\left(w_{C T}(\mathrm{AIC})=.03\right)$. Another way to assess the evidence in support of the force theory with respect to the causal model and mental model theories is to form evidence ratios in which the probability of the force model is normalized with respect to either the causal model (e.g., $w_{F T}$ (AIC) $\left./\left[w_{F T}(\mathrm{AIC})+w_{C T}(\mathrm{AIC})\right]\right)$ or mental model theory (Wagenmaker $\&$ Farrell, 2004). Such a ratio indicates that the probability that the force theory is to be preferred over the causal model theory is .95 , whereas the probability that the force theory is to be preferred over the mental model theory is .97. In summary, the AIC values support the force theory over the causal model and mental model theories.

One question raised by these findings concerns exactly why the force theory was more successful than the other theories in accounting for various correspondence relationships. In the case of the force theory and the causal model theory, the force theory's ability to rank possible correspondences proved decisive for its success in the present experiment. For example, the force theory predicts that $A$ prevents $B$ is better paraphrased by $A$ causes $\neg B$ and $\neg A$ allows $B$ than by $\neg A$ causes $B$ and $A$ allows $\neg B$; the causal model theory was not able to make this prediction. In the case of the force theory and the mental model theory, there were simply too many times that the force theory was able to predict the modal response, whereas the mental model theory was not.

\section{General Discussion}

Because nothing can be transmitted from an absence, the phenomenon of causation-by-omission has been viewed as a fatal flaw for process theories of causation (Menzies, 2006; Schaffer, 2000; Schulz et al., 2007; Woodward, 2006). The results from this article establish that this conclusion is incorrect. According to the force theory, causation-by-omission involves the actual or virtual removal of a force. Such removals entail a double prevention, that is, a situation in which a force that is preventing an event is removed by another force, thereby allowing the previously prevented event to occur and licensing the expression Lack of B allows/causes $C$. The force theory also proposes that the concept of ALLOW is understood in terms of double prevention, such that the double prevention described above can be interpreted as $A$ allows $C$.

The predictions of the force theory were supported by the results of four experiments. In Experiment 1, participants viewed animations depicting causal chains and chose from a list of sentences the expression that best described the animation. As predicted, participants chose ALLOW and CAUSE sentences as the best descrip- tion of the relationship between the first and third entities in the double prevention chains (i.e., A and C). Importantly, the relative proportion of ALLOW to CAUSE sentences varied with the magnitude of the patient vectors, just as predicted by the force theory. Also as predicted, when the two entities in question were the second and third entities in a double prevention (i.e., B and C), participants chose the sentences that described the effect as occurring from an omission. The results from Experiment 1 illustrate how causal relations can come about from the removal of a force, thus demonstrating that causal relations can occur even when a conserved quantitylike force or energy is not transmitted from the initial cause to the final effect. They also demonstrate an approach to causal representation that allows for causation-by-omission and also constrains it: Only omissions embedded in double preventions are likely to be construed of as possible causes. The theory thus offers a solution to the selection problem.

The results from Experiment 2 provided support for the force theory's prediction that when one or more CAUSE relations is added to a double prevention, the interpretation of the conclusion shifts from allowing-by-omission to causing-by-omission. Experiment 3 tested the force theory's proposal that transfer of energy or force between adjacent entities is not a necessary condition for causal relations. The motions of the objects involved may sometimes lead people to infer the realization of forces even before they occur. In Experiment 3, the mere anticipation of a force was sufficient to license a causal expression, suggesting that people's understanding of causal interactions is based not only on what occurs but also on what could have potentially occurred. Finally, with the evidence in support of the force theory in place, we compared the force theory with various dependency theories with respect to their predictions about how different types of causal statements are related to each other. In Experiment 4, participants were shown examples of the various types of causal expressions and asked to choose which sentence from a list of variant sentences was most similar in meaning to the initial expression. The correspondences chosen by people were better explained by the force theory than by any of the dependency theories. This result establishes not only that the force theory provides a viable account of causation by omission but also that causation by omission represents a significant challenge for dependency theories, despite what is often assumed.

\section{Why Is the Distinction Between CAUSE and ALLOW Important?}

In addition to providing an account of causation by omission, the force theory also offers an account of ALLOW. It could be argued that the goal of many dependency theories is not to provide an account of the meaning of causal expressions, but rather to explain causal learning and reasoning. In terms of reasoning, it may make little difference whether the relation connecting two events is ALLOW or CAUSE because both types of relations are generative; that is, in both cases, the probability of the effect is greater in the presence of the causal factor than in its absence. We submit, however, that the distinction between CAUSE and ALLOW is important for causal reasoning. In CAUSE relations, the strength of the effect is in some way proportional to the strength of the cause. For example, the amount of force exerted on the pedal of a bike is proportional to the bike's acceleration. 
Hence, in a CAUSE relation, we can infer the magnitude of the effect from the magnitude of the cause. In contrast, in ALLOW relations, there may be little or no relationship between the strength of the cause and the magnitude of the effect. For example, the rate at which water flows down a drain is independent of the magnitude of the force applied to release the plug. Thus, we cannot make inferences about the magnitude of the effect based on the magnitude of the cause. This is also true for causation by omission, which, like ALLOW relations, is based on the removal of a force. The ultimate result of causation by omission might be much greater than the magnitude of the cause. $^{7}$

\section{Causation That Might Seem to Resist a Force-Based Analysis}

Accounting for causation in terms of removals and virtual forces allows us to explain statements that might otherwise seem resistant to a force-based analysis. Consider, for example, the causal relation implied in the statement The ice caused him to slip. In this example, there is no apparent transmission of energy from the cause to the effect. Nevertheless, according to the force theory, causation can be instantiated when a CAUSE-type configuration results from the removal of a force via double prevention: Specifically, ice causes lack of friction and friction prevents slipping. The second PREVENT relation, friction prevents slipping, entails the absence of slipping; however, if ice causes lack of friction, then slipping will occur.

Another potentially problematic causal relation is implied in the statement A passing car caused a shadow in the room. Clearly, energy is not transmitted from the car to the wall in the room. Once again, though, this situation admits to a force-based analysis. Like the previous example, the scenario implies double prevention, $a$ passing car prevents sunlight, and sunlight prevents a shadow in the room, that leads to the conclusion A passing car caused a shadow in the room. Once the example is broken down, the connection to forces becomes more apparent. The first PREVENT relation, a passing car prevents sunlight, involves a straightforward application of forces: The car impedes the light by physically blocking its propagation. The second PREVENT relation, sunlight prevents the shadow from moving across the room, can be reanalyzed as sunlight prevents the absence of light in the room. Sunlight prevents the absence of light by hitting the wall and causing it to illuminate, which can be understood in terms of energy and force. It should be emphasized that the force theory does not insist on physically accurate mental models of the physical world. Clearly, our mental models of the physical world are often in error (Clement, 1983; McCloskey, 1983; McCloskey \& Kohl, 1983; McCloskey, Washburn, \& Felch, 1983). Rather, the force theory requires only that people generate mental models in a manner that uses forcelike quantities. On the basis of these quantities, the theory is able to offer an account of causal relations that do not at first glance appear to involve forces.

\section{Process Models, the Force Theory, and Exaptation}

We have classified the force theory as a process theory. Our use of the term process theory differs somewhat from the way this term has been used in philosophy (Dowe, 2007). In philosophy, a causal process is a propagation of a causal influence, which forms a world line (Salmon, 1984). When world lines overlap, the result is described as an intersection. One of the key issues for process theories is distinguishing actual from pseudoprocesses. A ball moving through the air constitutes an actual causal process, whereas the motion of a shadow is a pseudoprocess. The difference between actual and pseudocausal processes is that actual causal processes can carry a "mark" (Salmon, 1984), or, probably more accurately, they can carry a conserved property such as linear momentum, mass, or energy (Dowe, 2000).

In the present article, our interest was not in causal processes, per se, nor in distinguishing actual from pseudoprocesses, but rather in their interactions. In prior theories, both direct and indirect interactions must involve the transmission or exchange of a conserved quantity. According to the force theory, under certain conditions, which are spelled out by the theory, transmission or exchange of a conserved quantity is not necessary. This is a key difference between the force theory and other process theories. However, what all of these theories have in common is an ontological commitment to conserved quantities. In the case of the force theory, the commitment centers on forces. Forces may be special because qualitative representations of forces are still relatively informative. Forces have both direction and relative magnitude, whereas in the case of energy or mass, there can be differences in relative amount, but not differences in direction because these quantities are not vector quantities. Knowledge of the direction of influence, along with the relative magnitude of influence may be necessary for more complex forms of causal reasoning.

The force theory further assumes that the processes used to represent physical causation-vector addition and relation composition-are applied to represent and reason about causal relationships in the nonphysical domains. The observed role of virtual forces supports this assumption in demonstrating that forces need not be purely physical. This is yet another way in which the force theory differs from other process theories. The extension of mental operations for processing physical forces to the processing of nonphysical forces might be viewed as an exaptation. The mental machinery that evolved in people for processing physical forces may have been co-opted for causal representation and reasoning in the nonphysical domains. In the force theory, causal relations can be productively combined to produce infinitely many sequences of events. The representations of physical causation may provide the basis for recursive and combinatorial operations that support abstract thought. Although the content may change, the force theory predicts that the configuration of forces - and the recursive and combinatorial processes they entail—will remain the same.

\section{Forces in the Representation of Abstract Causal Relations}

We submit that the theoretical successes of the force theory stems from its commitment to forces, because without this commitment there would have been no reason to try to explain how causation might be grounded in the world, and hence, no reason to

\footnotetext{
${ }^{7}$ As shown by Frosch, Cowley, and Johnson-Laird (2009), the distinction between CAUSE and ALLOW also has implications for the assignment of responsibility and blame.
} 
try to explain causation by omission in terms of double prevention. But do we really think about abstract causal relations as if they were physical causal relations? For example, what forces were involved in Peter's failure to put gas in his car and the later stopping of the engine? From the perspective of the force theory, the story may be based on the double prevention Busyness prevented Peter from filling his gas tank and Peter's filling the tank prevents his car from running out of gas, then followed by the CAUSE relation Running out of gas causes the car to stop. As discussed earlier, the physical properties of the world constrain how a double prevention is realized, in particular, in a double prevention the second premise must be in place before the first premise can be applied. In the case of abstract causal relationships, this would imply a prevention that generally holds true. With respect to Peter's car, it is not Susan, Erik, the Pope, or the Queen of England that generally fills his gas tank, it is Peter. This is why the other people are not causally relevant to the event of running out of gas - they do not instantiate the PREVENT relation that must already be in place in order for the double prevention to go through. Given that only Peter tends to fill his gas tank, the removing force must be one that acts on Peter, perhaps busyness. Our solution to why Peter is considered the cause of his car stopping depends on the idea that Peter generally prevents his car from running out of gas. In effect, we have adopted the idea that causation by omission may depend on the notion of normality (Hart \& Honoré, 1985; McGrath, 2005). Importantly, the role of normality falls out naturally from the assumptions of the force theory, unlike in the case of dependency theories. The reason why it falls out of the force theory ultimately stems from the theory's commitment to physical force.

Our approach to double preventions also helps explain some of the intuition behind Cheng and Novick's (1991; see also Cheng, 1997) account of the difference between causers and enablers. Specifically, they propose that an enabler is a candidate causal factor that is constantly present in the focal set under consideration but that covaries with the effect in other possible focal sets. This approach associates ENABLE, but not CAUSE, with a default condition, the focal set that people are most likely to use in determining covariation. In our approach, the existence of a default condition is implied by the need for a preexisting PREVENT relationship.

\section{Conclusion}

According to the force theory, causation in the mind involves mental processes that reflect the way forces are joined in the world. It holds that people simulate the processes that produce causal relationships rather than simply specifying the dependencies that hold between one event or state and another. The results of the present research show that this kind of mental activity is not limited to situations in which the effects were produced from the presence of a force; rather, it can be extended to situations in which the effects are produced from the absence of forces. Moreover, the results from this research not only show how absences can be represented in forces but also provide evidence that when people think about negative causation, they use forcelike representations. Far from being a stumbling block, the phenomenon of causation by omission provides some of the strongest evidence that people reason about causation using forcelike units of cognition.

\section{References}

Ahn, W., \& Kalish, C. W. (2000). The role of mechanism beliefs in causal reasoning. In F. C. Keil \& R. A. Wilson (Eds.), Explanation and cognition (pp. 199-225). Cambridge, MA: MIT Press.

Aronson, J. L. (1971). On the grammar of 'cause'. Synthese, 22, 414-430.

Ashby, G. (1992). Multivariate probability distributions. In G. Ashby (Ed.), Multidimensional models of perception and cognition (pp. 1-34). Hillsdale, NJ: Erlbaum.

Autodesk. (2006). 3ds Max (Version 8) [Computer software]. San Francisco, CA: Author.

Barbey, A. K., \& Wolff, P. (2006). Causal reasoning from forces. Proceedings of the 28th Annual Conference of the Cognitive Science Society (p. 2439). Mahwah, NJ: Erlbaum.

Barbey, A. K., \& Wolff, P. (2007). Learning causal structure from reasoning. Proceedings of the 29th Annual Conference of the Cognitive Science Society (pp. 713-718). Mahwah, NJ: Erlbaum.

Barbey, A. K., \& Wolff, P. (2009). Composing causal relations in force dynamics. Manuscript submitted for publication.

Beebee, H. (2004). Causing and nothingness. In J. Collins, N. Hall, \& L. Paul (Eds.), Causation and counterfactuals (pp. 291-308). Cambridge, MA: MIT Press.

Beebee, H. (2005). P. 4.

Bullock, M., Gelman, R., \& Baillargeon, R. (1982). The development of causal reasoning. In W. Friedman (Ed.), The developmental psychology of time (pp. 209-255). London, England: Academic Press.

Chaigneau, S. E., \& Barbey, A. K. (2008). Assessing psychological theories of causal meaning and inference. In B. C. Love, K. McRae, \& V. M. Sloutsky (Eds.), Proceedings of the 30th Annual Conference of the Cognitive Science Society (pp. 1111-1116). Austin, TX: Cognitive Science Society.

Cheng, P. W. (1997). From covariation to causation: A causal power theory. Psychological Review, 104, 367-405.

Cheng, P. W., \& Novick, L. R. (1991). Causes versus enabling conditions. Cognition, 40, 83-120.

Cheng, P. W., \& Novick, L. R. (1992). Covariation in natural causal induction. Psychological Review, 99, 365-382.

Clement, J. (1983). A conceptual model discussed by Galileo and used intuitively by physics students. In D. Gentner \& A. L. Stevens (Eds.), Mental models (pp. 299-324). Hillsdale, NJ: Erlbaum.

Collins, J. (2000). Preemptive prevention. The Journal of Philosophy, 97, 223-234.

Craver, C. F. (2007). Explaining the brain: Mechanisms and the mosaic unity of neuroscience. Oxford, England: Oxford University Press.

Dowe, P. (2000). Physical causation. Cambridge, England: Cambridge University Press.

Dowe, P. (2001). A counterfactual theory of prevention and "causation" by omission. Australasian Journal of Philosophy, 79, 216-226.

Dowe, P. (2007). Causal processes. Stanford Encyclopedia of Philosophy. Retrieved from http://plato.stanford.edu/entries/causation-process/

Fair, D. (1979). Causation and the flow of energy. Erkenntnis, 14, 219250.

Foot, P. (1994). The problem of abortion and the doctrine of double effect. In B. Steinbock \& A. Norcross (Eds.), Killing and letting die (2nd ed., pp. 266-279). New York, NY: Fordham University Press. (Reprinted from Oxford Review, 5, 5-15, 1967.)

Freyd, J. J., \& Finke, R. A. (1984). A velocity effect for representational momentum. Bulletin of the Psychonomic Society, 23, 443-446.

Frosch, C. A., Cowley, M., \& Johnson-Laird, P. N. (2009). Causes, enablers and the law. Unpublished manuscript.

Godfrey-Smith, P. (in press). Causal pluralism. In H. Beebee, C. Hitchcock, \& P. Menzies (Eds.), Oxford handbook of causation.

Goldvarg, E., \& Johnson-Laird, P. (2001). Naive causality: A mental model theory of causal meaning and reasoning. Cognitive Science, 25, $565-610$. 
Hall, N. (2000). Causation and the price of transitivity. The Journal of Philosophy, 97, 198-222.

Hall, N. (2004). Two concepts of causation. In J. Collins, N. Hall, \& L. Paul (Eds.), Causation and counterfactuals (pp. 225-276). Cambridge, MA: MIT Press.

Hart, H. L., \& Honoré, A. (1985). Causation in the law. Oxford, England: Clarendon Press.

Hesslow, G. (1988). The problem of causal selection. In D. J. Hilton (Ed.), Contemporary science and natural explanation: Commonsense conceptions of causality (pp. 11-32). Brighton, Sussex, England: Harvester Press.

Hitchcock, C. (2001). The intransitivity of causation revealed in equations and graphs. The Journal of Philosophy, 98, 273-299.

Hubbard, T. L. (1995). Environmental invariants in the representation of motion: Implied dynamics and representational momentum, gravity, friction, and centripetal force. Psychonomic Bulletin \& Review, 2, 322-338.

Leslie, A. (1984). Spatiotemporal continuity and the perception of causality in infants. Perception, 13, 287-305.

Lewis, D. (1973). Causation. The Journal of Philosophy, 70, 556-567.

Lewis, D. (2000). Causation as influence. The Journal of Philosophy, 97, 182-197.

Lien, Y., \& Cheng, P. (2000). Distinguishing genuine from spurious causes: A coherence hypothesis. Cognitive Psychology, 40, 87-137.

Livengood, J., \& Machery, E. (2007). The folk probably don't think what you think they think: Experiments on causation by absence. Midwest Studies in Philosophy, XXXI, 107-127.

Lombrozo, T. (2009). Causal-explanatory pluralism: How intentions, functions, and mechanisms influence causal ascriptions. Manuscript under review.

Machamer, P., Darden, L., \& Craver, C. (2000). Thinking about mechanisms. Philosophy of Science, 67, 1-25.

Mackie, J. L. (1974). The cement of the universe. Oxford, England: Oxford University Press.

Mandel, D. R., \& Lehman, D. R. (1996). Counterfactual thinking and ascriptions of cause and preventability. Journal of Personality and Social Psychology, 71, 450-463.

Massaro, D. W. (2004). From multisensory integration to talking heads and language learning. In G. Calvert, C. Spence, \& B. E., Stein (Eds.), The handbook of multisensory processes (pp. 153-176). Cambridge, MA: MIT Press.

McCloskey, M. (1983). Naïve theories of motion. In D. Gentner \& A. L. Stevens (Eds.), Mental models (pp. 299-324). Hillsdale, NJ: Erlbaum.

McCloskey, M., \& Kohl, D. (1983). Naïve physics: The curvilinear impetus principle and its role in interactions with moving objects. Journal of Experimental Psychology: Learning, Memory, and Cognition, 9, 146-156.

McCloskey, M., Washburn, A., \& Felch, L. (1983). Intuitive physics: The straight-down belief and its origin. Journal of Experimental Psychology: Learning, Memory, and Cognition, 9, 636-649.

McGrath, S. (2003). Causation and the making/allowing distinction. Philosophical Studies, 114, 81-106.

McGrath, S. (2005). Causation by omission: A dilemma. Philosophical Studies, 123, 125-148.

McMahan, J. (1993). Killing, letting die, and withdrawing aid. Ethics, 103, $250-279$.

Menzies, P. (2006, November). A structural equations account of negative causation. Contributed papers of the Philosophy of Science Association 20th biennial Meeting. British Columbia, Vancouver, Canadas.
Pitt, M. A., \& Myung, J. (2002). When a good fit can be bad. Trends in Cognitive Sciences, 6, 421-425.

Psychology Software Tools, Inc. (2008). E-Prime (Version 2.0) [Computer software]. Pittsburgh, PA: Author.

Salmon, W. (1984). Scientific explanation and the causal structure of the world. Princeton, NJ: Princeton University Press.

Salmon, W. (1994). Causality without counterfactuals. Philosophy of Science, 61, 297-312.

Salmon, W. (1998). Causality and explanation. Oxford, England: Oxford University Press.

Schaffer, J. (2000). Causation by disconnection. Philosophy of Science, 67, 285-300.

Schulz, L., Kushnir, T., \& Gopnik, A. (2007). Learning from doing: Intervention and causal inference. In A. Gopnik \& L. Schulz (Eds.), Causal learning: Psychology, philosophy, and computation (pp. 67-85). Oxford, England: Oxford University Press.

Shultz, T. R. (1982). Rules of causal attribution. Monographs of the Society for Research in Child Development, 47(1, Serial No. 194), 1-51.

Sloman, S. (2005). Causal models: How people think about the world and its alternatives. Oxford, England: Oxford University Press.

Sloman, S. A., Barbey, A., \& Hotaling, J. (2009). A causal model theory of the meaning of cause, enable, and prevent. Cognitive Science, 33, 21-50.

Spellman, B. A., Kincannon, A. P., \& Stose, S. J. (2005). The relation between counterfactual and causal reasoning. In D. R. Mandel, D. J. Hilton, \& P. Catellani (Eds.), The psychology of counterfactual thinking (pp. 28-43). London, England: Routledge Research.

Spellman, B. A., \& Mandel, D. R. (1999). When possibility informs reality: Counterfactual thinking as a cue to causality. Current Directions in Psychological Science, 8, 120-123.

Talmy, L. (1988). Force dynamics in language and cognition. Cognitive Science, 12, 49-100.

Wagenmakers, E., \& Farrell, S. (2004). AIC model selection using Akaike weights. Psychonomic Bulletin \& Review, 11, 192-196.

Walsh, C. R., \& Sloman, S. (2005). The meaning of cause and prevent: The role of causal mechanism. Proceedings of the Twenty-Seventh Annual Conference of the Cognitive Science Society (pp. 2331-2336). Stresa, Italy. Hillsdale, NJ: Erlbaum.

Walsh, C. R., \& Sloman, S. (2009). The meaning of cause and prevent: The role of causal mechanism. Manuscript submitted for publication.

Wolff, P. (2007). Representing causation. Journal of Experimental Psychology: General, 136, 82-111.

Wolff, P., \& Song, G. (2003). Models of causation and the semantics of causal verbs. Cognitive Psychology, 47, 276-332.

Wolff, P., \& Zettergren, M. (2002). A vector model of causal meaning. In W. D. Gray \& C. D. Schunn (Eds.), Proceedings of the twenty-fourth annual conference of the Cognitive Science Society (pp. 944-949). Mahwah, NJ: Erlbaum.

Woodward, J. (2003). Making things happen. Oxford, England: Oxford University Press.

Woodward, J. (2006). Sensitive and insensitive causation. Philosophical Review, 115, 1-50.

Woodward, J. (2007). Interventionist theories of causation in psychological perspective. In A. Gopnik \& L. Schulz (Eds.), Causal learning: Psychology, philosophy, and computation (pp. 19-36). Oxford, England: Oxford University Press. 


\section{Appendix A}

Table A1

Magnitude of the Forces Acting on the Cars in Experiment 1

\begin{tabular}{cccccc}
\hline Forces & \multicolumn{1}{c}{$\mathrm{C} / \mathrm{C}$} & \multicolumn{1}{c}{$\mathrm{C} / \mathrm{P}$} & $\mathrm{P} / \mathrm{P}-1$ & $\mathrm{P} / \mathrm{P}-2$ & $\mathrm{P} / \mathrm{P}-3$ \\
\hline $\mathrm{A}$ & $5 \mathrm{~N}$, right & $5 \mathrm{~N}$, right & $4 \mathrm{~N}$, right & $4 \mathrm{~N}$, right & $5 \mathrm{~N}$, right \\
$\mathrm{B}$ & $3.5 \mathrm{~N}$, left & $4 \mathrm{~N}$, left & $4 \mathrm{~N}$, left & $2 \mathrm{~N}$, left & $3.5 \mathrm{~N}$, left \\
$\mathrm{C}$ & $2.5 \mathrm{~N}$, left & $2.5 \mathrm{~N}$, left & $2 \mathrm{~N}$, right & $2 \mathrm{~N}$, right & $3.5 \mathrm{~N}$, right \\
\hline
\end{tabular}

Note. $\mathrm{C} / \mathrm{C}=\mathrm{CAUSE} / \mathrm{CAUSE} ; \mathrm{C} / \mathrm{P}=\mathrm{CAUSE} / \mathrm{PREVENT} ; \mathrm{P} / \mathrm{P}=\mathrm{PREVENT} / \mathrm{PREVENT} ; \mathrm{N}=$ newtons .

Table A2

Magnitude of the Forces Acting on the Cars in Experiment 2

\begin{tabular}{lcll}
\hline Forces & Release-CAUSE & Release-ALLOW & Release-PREVENT \\
\hline $\mathrm{A}_{\text {Internal }}$ & Unknown & Unknown & Unknown \\
$\mathrm{A}_{\text {External }}$ & $4.5 \mathrm{~N}$, right & $4 \mathrm{~N}$, right & $4.5 \mathrm{~N}$, right \\
$\mathrm{B}$ & $4.5 \mathrm{~N}$, left & $4 \mathrm{~N}$, left & $4.5 \mathrm{~N}$, left \\
$\mathrm{C}$ & $0 \mathrm{~N}$ & $>4 \mathrm{~N}$, right (frictional) & $2 \mathrm{~N}$, right \\
\hline
\end{tabular}

Note. $\mathrm{N}=$ newtons.

Table A3

Magnitude of the Forces Acting on the Cars in Experiment 3

\begin{tabular}{llcccc}
\hline Forces & PREVENT & ALLOW-by-Absence1 & ALLOW-by-Absence2 & ALLOW-by-Holding & PREVENT-by-Holding \\
\hline $\mathrm{A}_{\text {Internal }}$ & Unknown & Unknown & Unknown & Unknown & Unknown \\
$\mathrm{A}_{\text {External }}$ & $8 \mathrm{~N}$, right & $8 \mathrm{~N}$, right & $5 \mathrm{~N}$, right & $4.5 \mathrm{~N}$, right & $4.5 \mathrm{~N}$, right \\
$\mathrm{B}$ & $3 \mathrm{~N}$, left & $3 \mathrm{~N}$, left & $4 \mathrm{~N}$, left & $4.5 \mathrm{~N}$, left & $4.5 \mathrm{~N}$, left \\
$\mathrm{C}$ & Unknown & & $2.5 \mathrm{~N}$, right & $2 \mathrm{~N}$, right & $0 \mathrm{~N}$ \\
\hline
\end{tabular}

Note. $\mathrm{N}=$ newtons. 
Table A4

Materials Used in Experiment 4

Sentence type

Causal sentence

A causes B

A allows B

A prevents $B$

$\neg \mathrm{A}$ causes $\mathrm{B}$

$\neg \mathrm{A}$ allows B

$\neg \mathrm{A}$ prevents $\mathrm{B}$

Blood clots cause strokes.

Cell phones cause car accidents.

Construction causes traffic congestion.

Erosion causes landslides.

Garlic causes bad breath.

Harmful radiation causes cancer.

Nerve damage causes pain.

Pollution causes global warming.

Tax cuts cause economic growth.

Tides cause currents.

Computers allow information exchange.

Democracy allows free speech.

E-mail allows communication.

Evolution allows adaptation.

Higher revenue allows for tax cuts.

Innovation allows modernization.

Investment allows expansion.

Microscopes allow observation.

Security flaws allow identity theft.

Statistics allow analysis.

Blizzards prevent travel.

Communication prevents misunderstanding.

Competition prevents inflation.

Deforestation prevents plant life.

Exercise prevents arthritis.

Milk prevents diabetes.

Nuts prevent blood clots.

Police prevent crime.

Spark detectors prevent explosions.

Tariffs prevent trade.

Absence of nicotine causes withdrawal.

Lack of education causes poverty.

Lack of light causes depression.

Lack of ozone allows harmful radiation.

Lack of zinc causes wilting.

Absence of democracy causes terrorism.

Absence of enzymes prevents metabolism.

Lack of fitness causes snoring.

Lack of knowledge causes confusion.

Lack of morals causes criminal behavior.

Lack of action allows salmon killing.

Lack of drainage allows fluid accumulation.

Lack of due process allows warrantless surveillance.

Lack of education allows easier political control.

Lack of smoke alarms allows fire spread.

Absence of regulations allows inappropriate use.

Lack of clouds allows sunshine.

Lack of organization allows exploitation.

Lack of traffic allows greater speeds.

Lack of wind allows dust settling.

Lack of expertise prevents adoption of new tools.

Lack of financial aid prevents graduation.

Lack of insurance prevents mental health care.

Lack of protein prevents lupus-like condition.

Lack of sleep prevents weight loss.

Lack of evidence prevents investigations.

Lack of iron prevents hemoglobin production.

Lack of publicity prevents reforms.

Lack of water prevents hygiene.

Lack of transparency prevents innovation. 
Table A4 (continued)

\begin{tabular}{ll}
\hline Sentence type & \multicolumn{1}{c}{ Causal sentence } \\
\hline A causes $\neg \mathrm{B}$ & Addictions cause lack of control. \\
& Broken bones cause lack of mobility. \\
& Chemical dependence causes lack of productivity. \\
& Communication barriers cause lack of understanding. \\
& Exercise causes lack of oxygen. \\
& Ignorance causes lack of skepticism. \\
& Laziness causes lack of work. \\
& Muscle relaxation causes an absence of stress. \\
& Pain causes lack of sleep. \\
& Surgery causes lack of sensation. \\
& Amateur radio allows no broadcasting. \\
& Black holes allow no escape. \\
A allows $\neg \mathrm{B}$ & College allows lack of self-discipline. \\
& Military regimes allow no dissent. \\
& Self-centeredness allows lack of empathy. \\
& Socialism allows no private property. \\
& Strict control allows no compromise. \\
& Technology allows an absence of friction. \\
& Unending workload allows no leisure. \\
& Vegan diets allow no animal products. \\
& Beverages prevent a lack of energy. \\
Consistency prevents lack of trust. \\
Education prevents the lack of skilled workers. \\
Judges prevent a lack of proper defense. \\
Proper diet prevents an absence of protein. \\
Reservoirs prevent lack of water. \\
Salt prevents the lack of iodine. \\
Transparent management prevents a lack of accountability. \\
Tree replacement prevents the lack of wood. \\
Water currents prevent lack of oxygen. \\
\hline $\mathrm{B}$ \\
\end{tabular}

\section{Appendix B}

\section{Correspondence Relationships for Four Dependency Theories}

Probabilistic contrast model. According to Cheng and Novick's (1992) probabilistic contrast model, a CAUSE relationship implies that the probability of effect in the presence of the cause, $\mathrm{P}(\mathrm{E} \mid \mathrm{C})$, is greater than the probability of the effect in its absence $\mathrm{P}(\mathrm{E} \mid \neg \mathrm{C})$, that is, $\mathrm{P}(\mathrm{E} \mid \mathrm{C})>\mathrm{P}(\mathrm{E} \mid \neg \mathrm{C})$. A PREVENT relationship implies the opposite, $\mathrm{P}(\mathrm{E} \mid \mathrm{C})<\mathrm{P}(\mathrm{E} \mid \neg \mathrm{C})$. In this theory, the statement $\neg C$ causes $E$ - causation by omission - would imply that the probability of $\mathrm{E}$ given $\neg \mathrm{C}, \mathrm{P}(\mathrm{E} \mid \neg \mathrm{C})$, is greater than the probability of $E$ given $C, P(E \mid C)$, that is, $P(E \mid \neg C)>P(E \mid C)$, which is the same inequality as that for PREVENT. In other words, the criterion for the claim $\neg A$ causes $B$ is the same as for $A$ prevents $B$. Interestingly, the probabilistic contrast model also predicts that CAUSE NOT implies PREVENT. According to the probabilistic model, CAUSE NOT implies that the probability of the absence of the effect, $\mathrm{P}(\neg \mathrm{E} \mid \mathrm{C})$, is greater in the presence of the cause than in the absence of the cause, $\mathrm{P}(\neg \mathrm{E} \mid \neg \mathrm{C})$, that is, $\mathrm{P}(\neg \mathrm{E} \mid \mathrm{C})>\mathrm{P}(\neg \mathrm{E} \mid \neg \mathrm{C})$. This inequality entails $\mathrm{P}(\mathrm{E} \mid \mathrm{C})<\mathrm{P}(\mathrm{E} \mid \neg \mathrm{C})$, which entails the same inequality that defines PREVENT relations. The inequality asso- ciated with the claim $C$ prevents $\neg E$ would be $\mathrm{P}(\neg \mathrm{E} \mid \mathrm{C})<$ $\mathrm{P}(\neg \mathrm{E} \mid \neg \mathrm{C})$, which entails $\mathrm{P}(\mathrm{E} \mid \mathrm{C})>\mathrm{P}(\mathrm{E} \mid \neg \mathrm{C})$, the same inequality associated with $C$ causes $E$. Similarly, the claim $\neg C$ prevents $E$ would be associated with the inequality $\mathrm{P}(\mathrm{E} \mid \neg \mathrm{C})<\mathrm{P}(\mathrm{E} \mid \neg \neg \mathrm{C})$, and thus $\mathrm{P}(\mathrm{E} \mid \mathrm{C})>\mathrm{P}(\mathrm{E} \mid \neg \mathrm{C})$, again the same inequality associated with the claim $C$ causes $E$. In summary, according to the probabilistic contrast model, $A$ causes $\neg B$ implies $A$ prevents $B$ to the same degree that $\neg A$ causes $B$ implies $A$ prevents $B$. Furthermore, $A$ prevents $\neg B$ and $\neg A$ prevents $B$ both imply $A$ causes $B$.

Counterfactual theories. Counterfactual theories of causation make a related set of predictions (Lewis, 1973). As noted earlier, $A$ causes $B$ holds if it is the case that if $\mathrm{A}$ had not occurred, $B$ would not have occurred. A counterfactual criterion can presumably be extended to PREVENT relations (see Dowe, 2001). A counterfactual criterion for PREVENT would be the same as the CAUSE criterion, except for the valence of the outcome. Specifically, as suggested by Walsh and Sloman (2009), A prevents $B$ holds if it is the case that if A had not occurred, B would have 
occurred. A counterfactual criterion for $A$ causes $\neg B$ would presumably be the same as well, implying that $A$ prevents $B$ and $A$ causes $\neg B$ have the same meaning (Walsh \& Sloman, 2009). The meaning of the causal claim $\neg A$ causes $B$ would presumably map onto the conditional if $A$ had occurred, $B$ would not have occurred (McGrath, 2005). Importantly, though, if $A$, then not $B$ does not imply if not $A$, then $B$; hence, on a counterfactual account, $\neg A$ causes $B$ does not have the same meaning as $A$ causes $\neg B$ or $A$ prevents $B$.

Mental model theory. A third type of outcome theory is Goldvarg and Johnson-Laird's (2001) mental model theory. The model theory goes beyond other theories in not only characterizing CAUSE and PREVENT but also distinguishing these two notions from ALLOW. According to the mental model theory, the notions of CAUSE, ALLOW, and PREVENT are associated with different combinations of possible co-occurrences. The sets of possible co-occurrences associated with CAUSE, ALLOW, and PREVENT are shown in the second column of Table B1. For example, a CAUSE relation is associated with a set of co-occurrences in which $\mathrm{A}$ is present and $\mathrm{B}$ is present, $\mathrm{A}$ is absent and $\mathrm{B}$ is present, and $\mathrm{A}$ is absent and B is present. Applying NOT to the antecedent or consequent flips the states of affairs of the antecedents and consequents (respectively) in all of the possible co-occurrences.

As with the previous outcome theories of causation, the mental model theory predicts various correspondences between negated and nonnegated causal relations by virtue of common sets of co-occurrences. For example, as shown in Table B1, the theory predicts that $A$ prevents $B$ and $A$ causes $\neg B$ should be paraphrases because they share the same set of co-occurrences and, hence, the same truth conditions. In addition to this pair of correspondences, the theory predicts the following correspondence relationships: $A$ causes $B$ and $A$ prevents $\neg B$, A prevents $B$ and $\neg A$ allows $B, \neg A$ causes $B$ and $A$ allows $\neg B$, and $\neg A$ prevents $B$ and $A$ allows $B$.

In generating predictions for the mental model theory, we relied exclusively on the correspondences entailed by the truth conditions specified in Table B1. However, according to the mental model theory, people only represent the pairs of possible co-occurrences associated with various causal relations. For certain pairs of causal relations, the truth conditions, as specified in Table B1, are the same, but the possible co-occurrences (i.e., mental models) that are explicitly represented differ. As a consequence, whereas two relations may have the same truth conditions, it may not be intuitively obvious they are paraphrases of each other. Thus, it is possible that some of the predictions we have drawn for the mental model theory might not necessarily follow.

Causal model theory. A fourth type of dependency theory is represented by causal Bayesian network theories of causation. In causal Bayesian networks, variables are connected to one another with "arcs," as in A $\rightarrow$ B. Each arc is associated with a set of conditional probabilities in conjunction with assumptions about the effect of actual or hypothetical interventions (Schulz et al., 2007; Sloman, 2005; Woodward, 2003, 2007). A recent account called the causal model theory shows how a Bayesian network approach to causation might be applied to the representation of CAUSE, ALLOW, and PREVENT, including causation by omission (Sloman et al., 2009). Sloman et al. (2009) framed their theory in terms of structural equations, which represent a particular way of instantiating a graph with arrows. For example, the graph $\mathrm{A} \rightarrow$ $\mathrm{B}$ is instantiated in a structural equation such as B := A (Sloman et al., 2009; see also Hitchcock, 2001). According to their theory, the claim $A$ causes $B$ would be represented by the structural equation $\mathrm{B}:=\mathrm{A}$. The concept of ALLOW is associated with a different structural equation; for example, the claim $A$ allows $B$ would be represented as $\mathrm{B}:=\mathrm{A}$ and $\mathrm{X}$, in which the variable $\mathrm{X}$ is an accessory variable. Sloman et al. (2009) speculate that the concept of PREVENT is vaguer than CAUSE or ALLOW and, as a consequence, may be represented by several structural equations. On their account, the claim $A$ prevents $B$ could be represented by either $\mathrm{B}:=\neg \mathrm{A}$, B $:=\neg \mathrm{A}$ and $\mathrm{X}$, or $\mathrm{B}:=\neg \mathrm{A}$ and $\neg \mathrm{X}$.

As with the other theories of causation, the causal model theory predicts various correspondences between negated and nonnegated causal relations. According to the causal model theory, a claim such as $\neg A$ causes $B$ would be represented by negating the $\mathrm{A}$ variable in the structural equation associated with CAUSE relations (Sloman et al., 2009); specifically, it would be represented by the equation $\mathrm{B}:=\neg \mathrm{A}$. As noted above, PREVENT relations can also be represented by $\mathrm{B}:=\neg \mathrm{A}$; hence, the causal model theory predicts that claims such as $\neg A$ causes $B$ can be paraphrased as $A$ prevents $B$. A claim such as $A$ causes $\neg B$ would be represented by negating the $B$ variable in the CAUSE structural equation, leading to $\neg \mathrm{B}:=\mathrm{A}$. Importantly, the truth conditions for $\neg \mathrm{B}:=\mathrm{A}$ are the same as those for $\mathrm{B}:=\neg \mathrm{A}$ (e.g., if $\mathrm{A}=$ TRUE, then $\mathrm{B}=$ FALSE). As a consequence, in terms of truth conditions, the causal model theory predicts that CAUSE NOT relations can be interpreted as NOT CAUSE relations and that both of these relations can be paraphrased as PREVENT relations. To the extent that there are

Table B1

Possible Co-Occurrences Associated With the Concepts CAUSE, ALLOW, PREVENT and Various Derivatives Through Negation

\begin{tabular}{|c|c|c|c|c|c|}
\hline Basic relation & Co-occurrence & Antecedent negated & Co-occurrence & Consequent negated & Co-occurrence \\
\hline CAUSE & $\begin{array}{l}\mathrm{a} \mathrm{b} \\
\neg \mathrm{a} \mathrm{b} \\
\neg \mathrm{a} \neg \mathrm{b}\end{array}$ & $\neg$ A_CAUSE & $\begin{array}{l}\neg \mathrm{a} b \\
\mathrm{a} b \\
\mathrm{a} \neg \mathrm{b}\end{array}$ & CAUSE $\neg$ B & $\begin{array}{l}\mathrm{a} \neg \mathrm{b} \\
\neg \mathrm{a} \neg \mathrm{b} \\
\neg \mathrm{a} \mathrm{b}\end{array}$ \\
\hline ALLOW & $\begin{array}{l}\mathrm{a} b \\
\mathrm{a} \neg \mathrm{b} \\
\neg \mathrm{a} \neg \mathrm{b}\end{array}$ & $\neg$ A_ALLOW & $\begin{array}{l}\neg \mathrm{a} b \\
\neg \mathrm{a} \neg \mathrm{b} \\
\mathrm{a} \neg \mathrm{b}\end{array}$ & ALLOW $\neg \mathrm{B}$ & $\begin{array}{l}\mathrm{a} \neg \mathrm{b} \\
\mathrm{a} \mathrm{b} \\
\neg \mathrm{a} \mathrm{b}\end{array}$ \\
\hline PREVENT & $\begin{array}{l}\mathrm{a} \neg \mathrm{b} \\
\neg \mathrm{a} \mathrm{b} \\
\neg \mathrm{a} \neg \mathrm{b}\end{array}$ & $\neg$ A_PREVENT & $\begin{array}{l}\neg \mathrm{a} \neg \mathrm{b} \\
\mathrm{a} \mathrm{b} \\
\mathrm{a} \neg \mathrm{b}\end{array}$ & PREVENT $\neg \mathrm{B}$ & $\begin{array}{l}\mathrm{ab} \\
\neg \mathrm{a} \neg \mathrm{b} \\
\neg \mathrm{a} \mathrm{b}\end{array}$ \\
\hline
\end{tabular}

Note. $\neg \mathrm{A}=$ lack of antecedent; $\neg \mathrm{B}=$ lack of consequent. 
any asymmetries between these relations and PREVENT relations, PREVENT relations should be more easily paraphrased as NOT CAUSE relations than CAUSE NOT relations. As in several of the theories, the causal model theory predicts that claims such as $\neg A$ allows $B$ entail the claim $A$ prevents $B$, because the structural equation for $\neg A$ allows $B$, B : $=\neg \mathrm{A}$ and $\mathrm{X}$, is one of the equations associated with claims such as $A$ prevents $B$. The claim $\neg A$ prevents $B$ could be associated with several structural equations, including $\mathrm{B}:=\neg(\neg \mathrm{A})$, which would reduce to $\mathrm{B}:=\mathrm{A}$, that is, a simple CAUSE relation. The claim $\neg A$ prevents $B$ could also be associated with an equation such as $\mathrm{B}:=\neg(\neg \mathrm{A})$ and $\mathrm{X}$ or B $:=$ $\neg(\neg \mathrm{A})$ and $\neg \mathrm{X}$. The equation $\mathrm{B}:=\neg(\neg \mathrm{A})$ and $\mathrm{X}$ reduces to $\mathrm{B}:=$ $\mathrm{A}$ and $\mathrm{X}$; thus, the causal model theory predicts that the claim not $A$ prevents $B$ can be paraphrased as $A$ allows $B$ or $A$ causes $B$. The claim $A$ prevents not $B$ is also associated with several possible equations: $\neg \mathrm{B}:=\neg \mathrm{A}, \neg \mathrm{B}:=\neg \mathrm{A}$ and $\mathrm{X}$, or $\neg \mathrm{B}:=\neg \mathrm{A}$ and $\neg \mathrm{X}$, which entail the equations $\mathrm{B}:=\mathrm{A}, \mathrm{B}:=\mathrm{A}$ and $\neg \mathrm{X}$, and $\mathrm{B}:=\mathrm{A}$ and $\mathrm{X}$. The causal model theory predicts, then, that the claim $\neg A$ prevents $B$ may be paraphrased as either $A$ causes $B$ or $A$ allows $B$. Finally, the claim $A$ allows $\neg B$ would be associated with the equation $\neg \mathrm{B}:=\mathrm{A}$ and $\mathrm{X}$, which is compatible with the same truth conditions as $\mathrm{B}:=\neg \mathrm{A}$ and $\neg \mathrm{X}$, one of the equations associated with PREVENT. Thus, the model theory predicts that it should be possible to paraphrase $A$ allows $\neg B$ as $A$ prevents $B$.

Received March 4, 2009

Revision received September 29, 2009

Accepted September 30, 2009

\section{Members of Underrepresented Groups: Reviewers for Journal Manuscripts Wanted}

If you are interested in reviewing manuscripts for APA journals, the APA Publications and Communications Board would like to invite your participation. Manuscript reviewers are vital to the publications process. As a reviewer, you would gain valuable experience in publishing. The P\&C Board is particularly interested in encouraging members of underrepresented groups to participate more in this process.

If you are interested in reviewing manuscripts, please write APA Journals at Reviewers@apa.org. Please note the following important points:

- To be selected as a reviewer, you must have published articles in peer-reviewed journals. The experience of publishing provides a reviewer with the basis for preparing a thorough, objective review.

- To be selected, it is critical to be a regular reader of the five to six empirical journals that are most central to the area or journal for which you would like to review. Current knowledge of recently published research provides a reviewer with the knowledge base to evaluate a new submission within the context of existing research.

- To select the appropriate reviewers for each manuscript, the editor needs detailed information. Please include with your letter your vita. In the letter, please identify which APA journal(s) you are interested in, and describe your area of expertise. Be as specific as possible. For example, "social psychology" is not sufficient-you would need to specify "social cognition" or "attitude change" as well.

- Reviewing a manuscript takes time (1-4 hours per manuscript reviewed). If you are selected to review a manuscript, be prepared to invest the necessary time to evaluate the manuscript thoroughly. 